\title{
Top pair and single top production in ATLAS
}

Mederica Fabbri ‥ T- intant On behalf of the ATLAS collaboration Lomoniosov18, Moscal

I.*. and

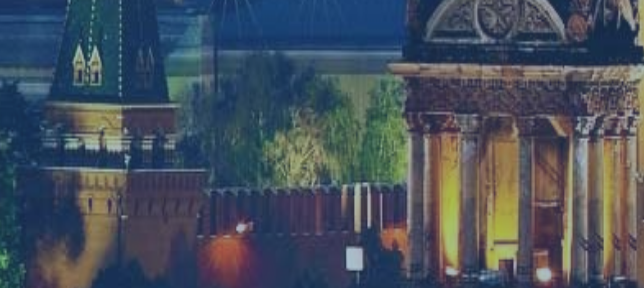

"y 


\section{Why top quark and $\sigma(t)$ and $\sigma(t \mathfrak{t})$ ?}

Elementary particle with the largest mass:

World average: arXiv:1403.4427

$$
m_{t}=173.34 \pm 0.27^{\text {(stat) }} \pm 0.71^{\text {(syst) }} \mathrm{GeV}
$$

$\mathrm{m}_{\mathrm{t}} \sim$ electroweak symmetry breaking scale The only quark that decays before hadronize:

- Direct access to Wt coupling

- Direct access to "free quark" properties

Stringent test of the latest theoretical calculation (NNLO, NLO +NNLL)

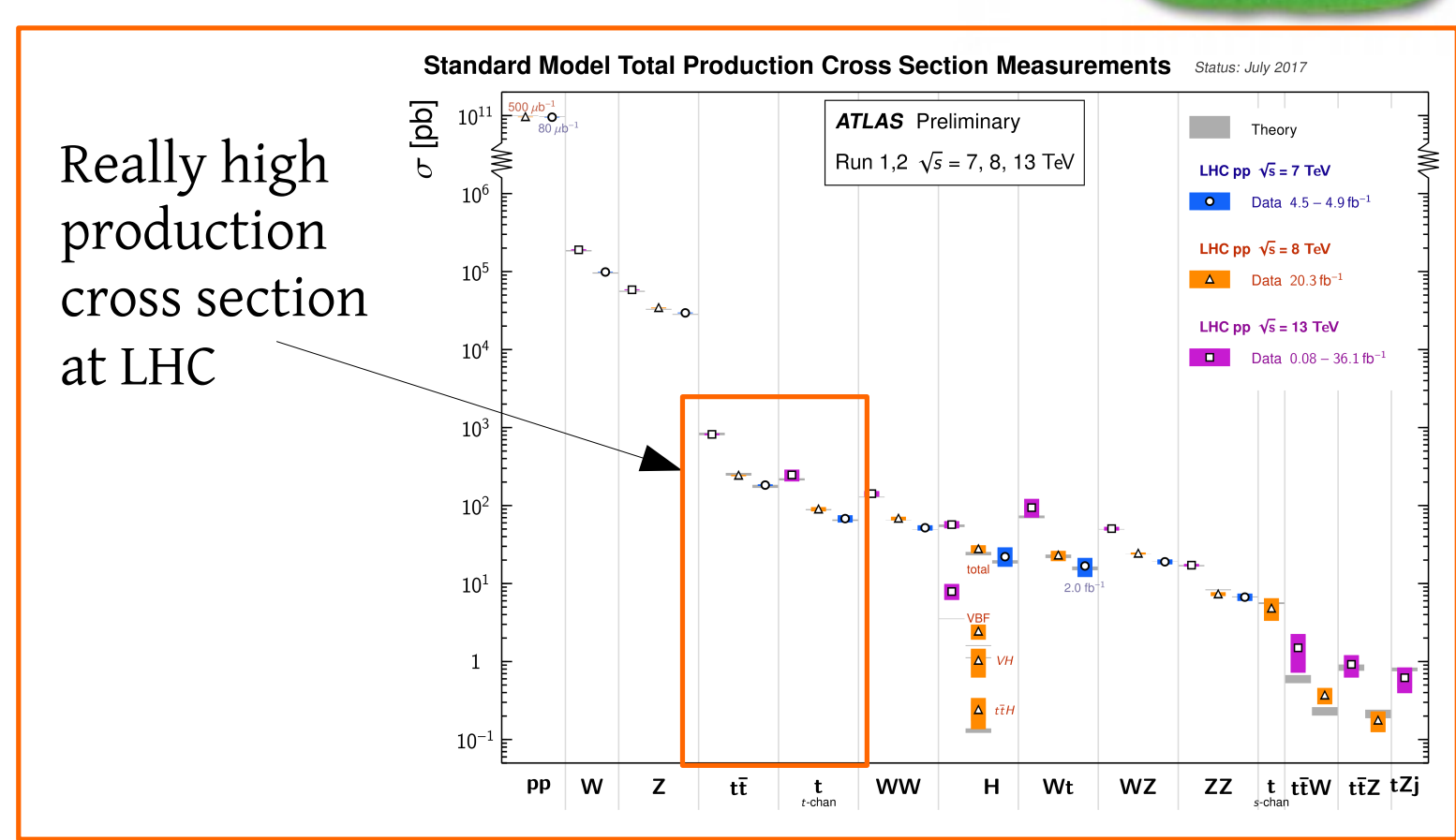

Involved in many BSM theories Hint of new physics:

- Observed in modification of $\sigma(t \bar{t})$ and $\sigma(t)$ shape

- Observed in a variation of coupling wrt SM expectations

Main background to many searches (graviton, Z' and stop searches, t $\overline{\mathrm{t}} \mathrm{H} . .$. ), dominated from $\sigma(t \bar{t})$ theoretical uncertainties

Precise measurement input to:

- MC tuning

ATL-PHYS-PUB-2016-020

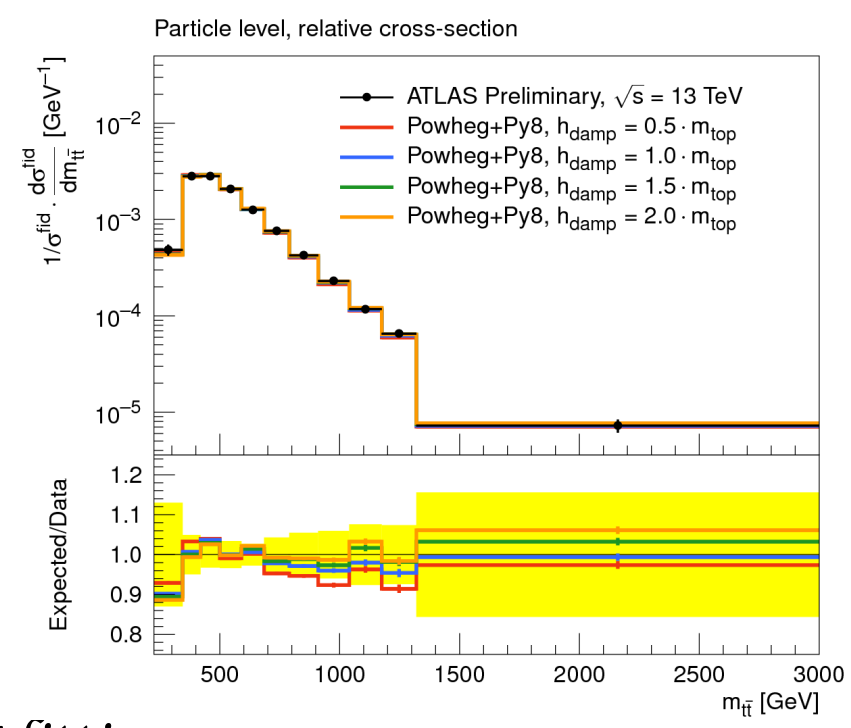

PDF fitting:

$\mathrm{t} \overline{\mathrm{t}} \rightarrow$ gluons, $\mathrm{t}(\overline{\mathrm{t}}) \rightarrow \mathrm{b}$, light quark PDFs 


\section{$\mathrm{t} \bar{t}$ production and decay @ LHC}

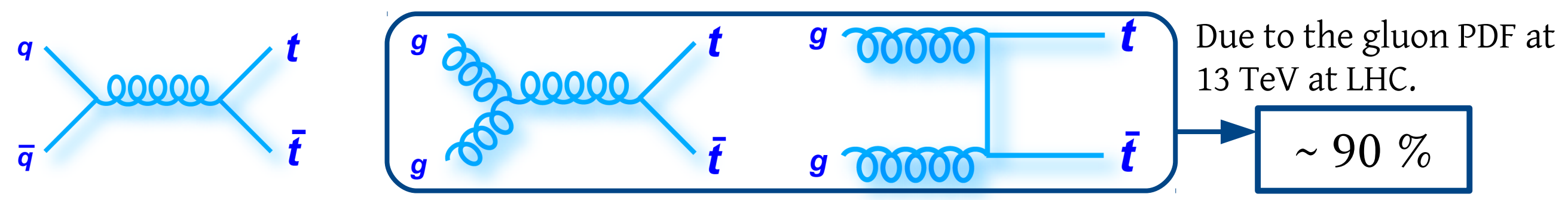

$\mathrm{t} \rightarrow \mathrm{Wb}(\mathrm{BR} \sim 99 \%)$
Analysis channel: Final states and analysis channels depend on the decay of the two $\mathrm{W}$ bosons.

\begin{tabular}{|c|c|c|}
\hline$\sqrt{ }$ & $8 \mathrm{TeV}$ & $13 \mathrm{TeV}$ \\
\hline$\sigma(\mathrm{t} \overline{\mathrm{t}})(\mathrm{pb})$ & $253_{-15}^{+13}$ & $832_{-46}^{+40}$ \\
\hline
\end{tabular}

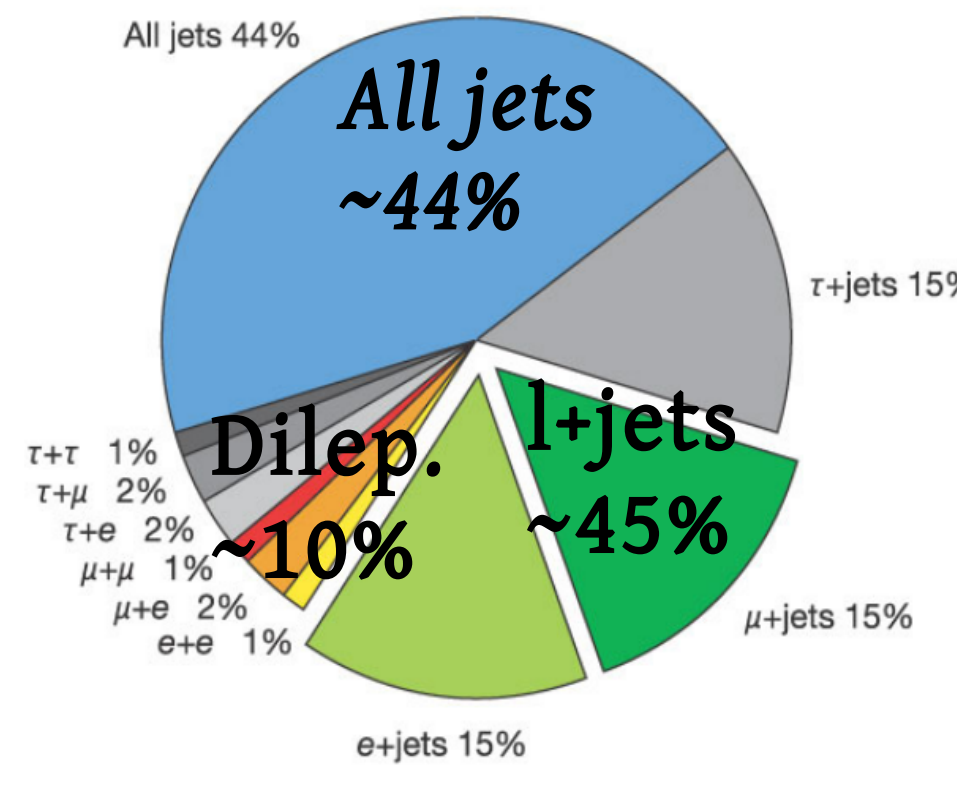

So many interesting measurements, also in 2017!!!

\begin{tabular}{|c|c|c|c|c|}
\hline & $\sqrt{ } \mathrm{s}=$ & dilepton & $1+$ jets & All jets \\
\hline \multirow{3}{*}{$\sigma(t \bar{t})$} & \multirow[t]{2}{*}{$8 \mathrm{TeV}$} & \multirow[t]{2}{*}{$\begin{array}{l}\text { Eur.Phys.J. C74 (2014) no } \\
.10,3109\end{array}$} & $\begin{array}{l}\quad \text { tau+jets: } \\
\text { Phys. Rev. D } 95 \text { (2017) } 07 \\
\text { P2003 }\end{array}$ & \\
\hline & & & ATLAS-CONF-2017-054 & \\
\hline & $13 \mathrm{TeV}$ & $\begin{array}{l}\text { Phys.Lett. B761 (2016) } 1 \\
\text { 36-157 }\end{array}$ & ATLAS-CONF-2015-049 & \\
\hline \multirow{3}{*}{$\frac{\mathrm{d} \sigma(\mathrm{tt})}{\mathrm{dx}}$} & \multirow{2}{*}{$8 \mathrm{TeV}$} & PhysRevD.94.092003 & $\begin{array}{l}\text { Resolved: } \\
\text { Eur. Phys. J. C76 (2016) } 538\end{array}$ & \\
\hline & & $\begin{array}{c}\text { Including } m^{t:} \\
\text { ATLAS-CONF-2017-044 }\end{array}$ & $\begin{array}{c}\text { Boosted: } \\
\text { Phys. Rev. D93 (2016) } 032009\end{array}$ & \\
\hline & $13 \mathrm{TeV}$ & $\begin{array}{l}\text { Eur. Phys. J. C77 (2016 } \\
\text { ) } 299\end{array}$ & $\begin{array}{l}\text { Resolved \& Boosted: } \\
\text { CERN-EP-2017-058 }\end{array}$ & $\begin{array}{l}\text { Boosted: } \\
\text { ATLAS-CONF-2016-100 }\end{array}$ \\
\hline
\end{tabular}




\section{$\sigma(t \mathfrak{t})$ in $\tau+j$ ets @ $8 \mathrm{TeV}$}

Good cross check to the cross section measurement in other channels. BSM mass and flavour dependent couplings expected to enhance $t \rightarrow \tau v_{\tau} b$ (SUSY, 2HDM includes $\mathrm{t} \rightarrow \mathrm{Hq} \rightarrow \tau \tau q)$

\section{Analysis strategy:}

-Final state: $\mathrm{t} \overline{\mathrm{t}} \rightarrow \mathrm{bbqq} \tau v_{\tau}, \tau$ decaying to hadrons

O $\tau$ identification using boosted decision tree (BDT), depending on the number of charged particles in $\tau$ decay

Background identification:

MC Estimate: events containing a real $\tau$ (Diboson, Single Top, $\mathrm{V}+\mathrm{jets}$ ) and with mis-identification of $e, \mu$ (mainly from $\mathrm{t} \overline{\mathrm{t}}$ decay)

Data driven: mis-identified jets from gluons or quarks Result:

$$
\begin{gathered}
\sigma(p p \rightarrow t \bar{t}+X)=\frac{N_{\mathrm{data}}-N_{\mathrm{bkg}-\mathrm{non} t \bar{t}}}{\mathrm{BR} \times\left(\epsilon_{t \bar{t}}-\epsilon_{\mathrm{FF}-t \bar{t}}\right) \times \int \mathcal{L}(t) d t} \\
\sigma_{t \bar{t}}=239 \pm 4(\text { stat }) \pm 28 \text { (syst) } \pm 5(\text { lumi }) \mathrm{pb} \\
\text { Mainly modelling, b-tagging }
\end{gathered}
$$

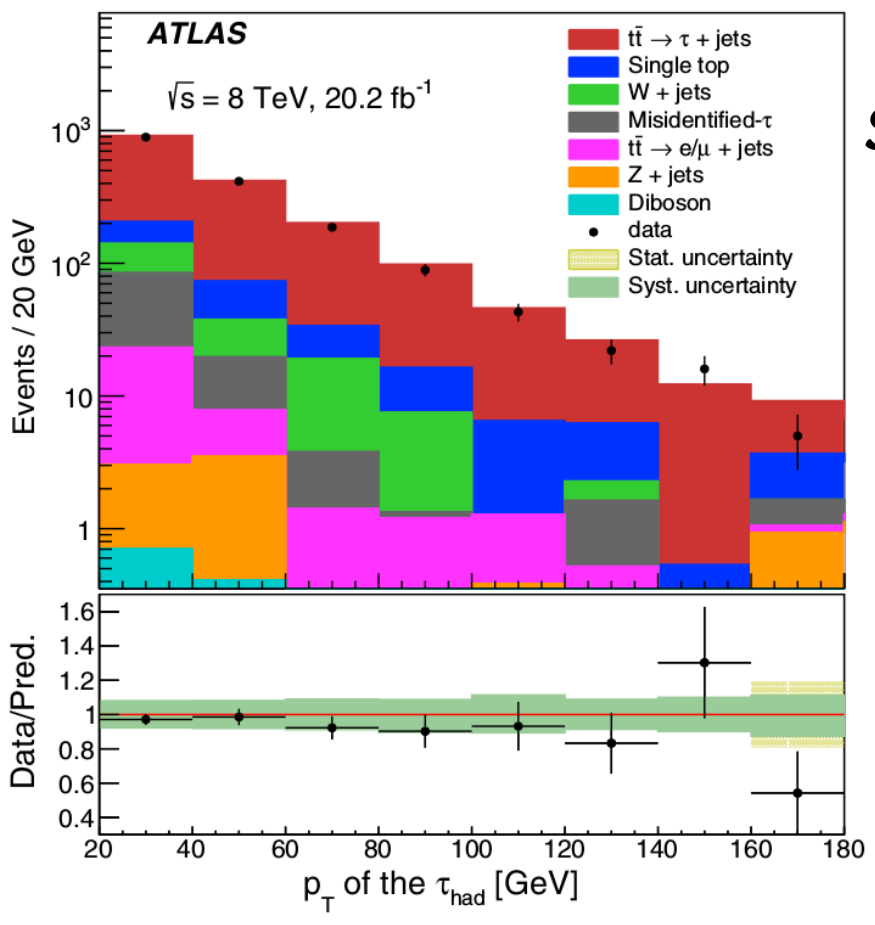

Signal purity $80 \%$

\begin{tabular}{lc}
\hline Observed data & 1678 \\
\hline Expected SM background & $1751 \pm 42$ \\
$S_{\mathrm{obs}(\exp )}^{95}$ & $446\left(444_{-21}^{+40}\right)$ \\
$\langle\epsilon \sigma\rangle_{\mathrm{obs}(\exp )}^{95}[\mathrm{fb}]$ & $22\left(22_{-1}^{+2}\right)$ \\
\hline \hline
\end{tabular}

Good agreement with the SM expectations and other channel measurements.

- Set a limit on the existence of the flavour changing neutral current process: $t \rightarrow q H \rightarrow q \tau \tau$ 


\section{$\sigma(t \bar{t})$ in l+jets @ $8 \mathrm{TeV}$ using NN}
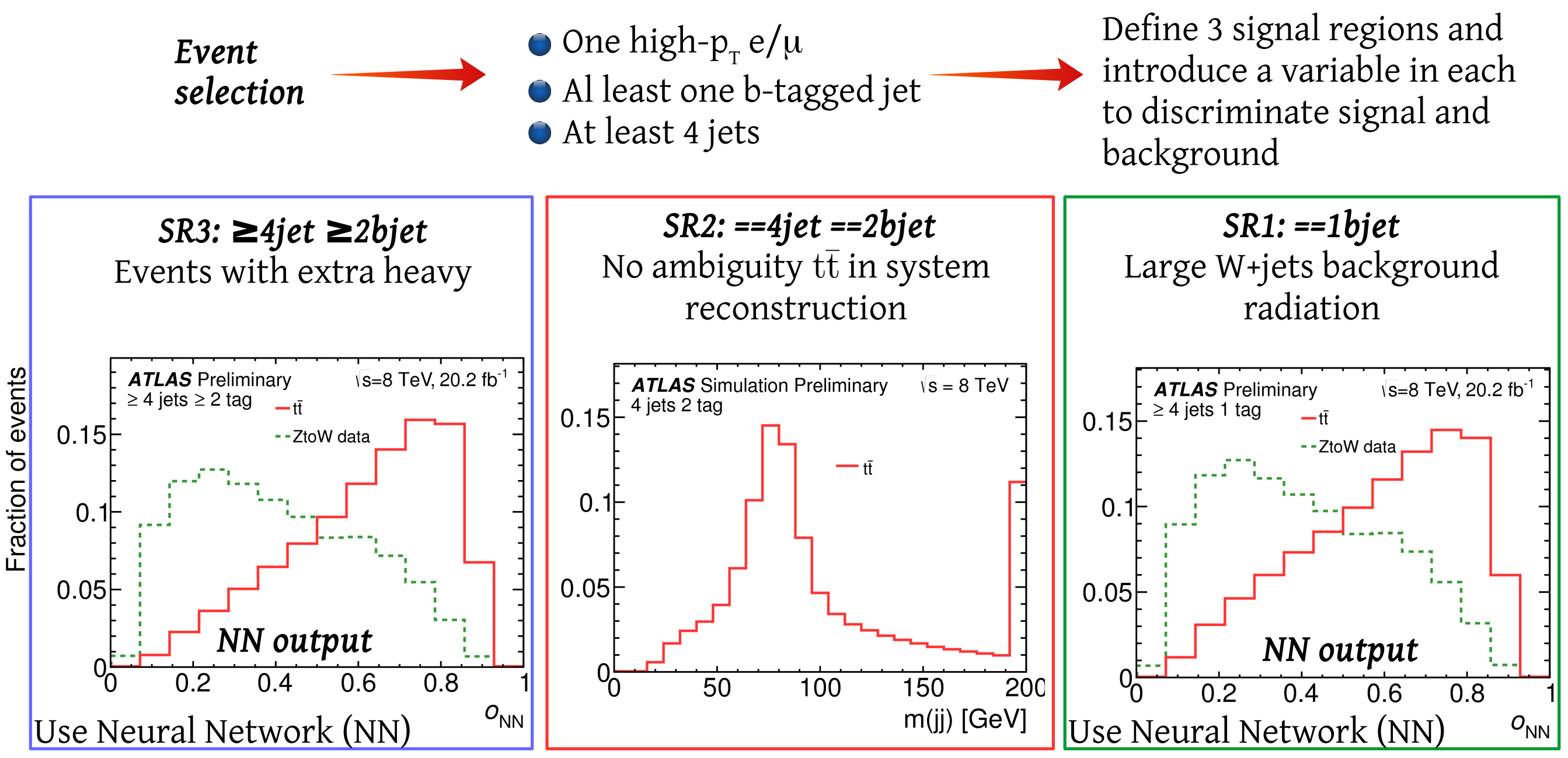

Binned likelihood fit on the three signal regions to extract $\sigma(t \bar{t})$ :

$$
\sigma_{t \bar{t}}=248.3 \pm 0.7 \text { (stat.) } \pm 13.4 \text { (syst.) } \pm 4.7 \text { (lumi.) } \mathrm{pb}
$$

- Uncertainty constrained in the fit as nuisance parameters, dominant Jet Energy scale (JES) and t $\overline{\mathrm{t}}$ modelling

- Good agreement with the SM expectations 


\section{Differential measurements}

- Different distributions are sensitive to different aspects of $t \bar{t}$ production

$\rightarrow$ Very stringent test of QCD calculations

$\rightarrow \mathrm{MC}$ tuning, PDF fitting

- Identify presence of BSM in shape modifications

- Handle the limited resolution of the detector and correct for it

$\rightarrow$ Use MC to evaluate a response matrix that accounts for mis-reconstruction of kinematic quantities.

Depending on the way the corrections are evaluated, the measurement can be performed at:

CERN-EP-2017-058

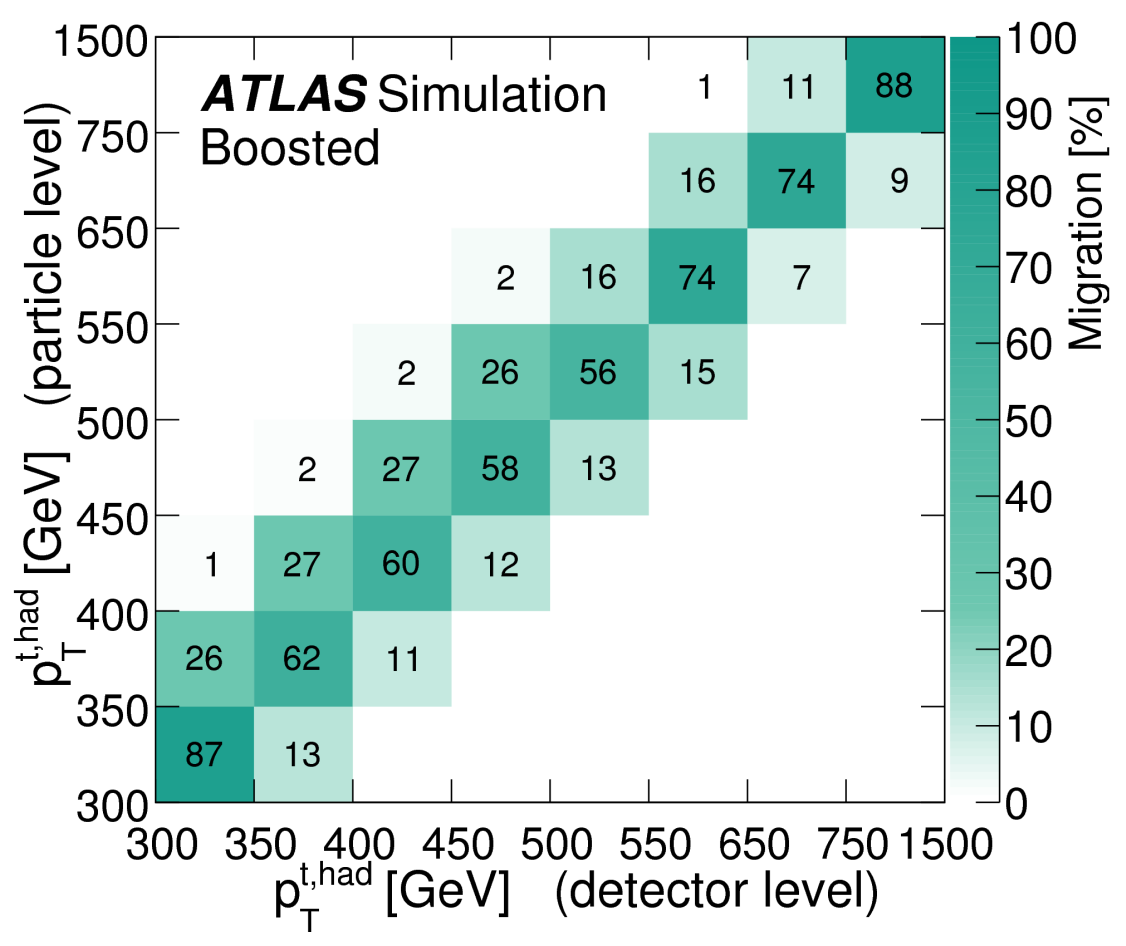

\section{Particle Level:}

- Truth: "objects"(leptons, jets) reconstructed from stable particles, before detector interactions.

- Apply on the "objects" fiducial cuts, similar to the reconstruction cuts

$\rightarrow$ Smaller modelling uncertainties (smaller extrapolation)

$\rightarrow$ Reduce dependencies from generators

\section{Parton level:}

- Truth: $\bar{t}$ and $t$, after radiations before decay.

- Measurement extrapolated to the full phase space

- Compare the result with the latest theoretical calculations not available at particle level 


\section{$\mathrm{d \sigma}(\mathrm{t} \overline{\mathrm{t}})$ in e $\mu$ channel @ 8TeV}

Measurement of the $\sigma(\mathfrak{t} \mathfrak{t})$ in e $\mu$ channel as a function of the lepton's kinematic property.

- Pre-selection requires e $\mu$ with opposite sign (OS) $\rightarrow$ Events afterward divided depending on the number of reconstructed b-tagged jets: N1,N2.

Cross section extracted bin-by-bin (i) from:

$$
\begin{aligned}
& N_{1}^{i}=L \sigma_{t \bar{t}}^{i} G_{e \mu}^{i} 2 \epsilon_{b}^{i}\left(1-C_{b}^{i} \epsilon_{b}^{i}\right)+N_{1}^{i, \mathrm{bkg} g} \\
& N_{2}^{i}=L \sigma_{t \bar{t}}^{i} G_{e \mu}^{i} C_{b}^{i}\left(\epsilon_{b}^{i}\right)^{2}+N_{2}^{i, \mathrm{bkg}},
\end{aligned}
$$

Binned efficiency and migration correction evaluated from MC.

\section{Results:}

\section{Differential cross} section

- Main uncertainty: statistics and background determination

- Good agreement with $\mathrm{SM}$, dependence wrt the lepton $\mathrm{p}_{\mathrm{T}}$ improved using HERAPDF and NNLO predictions.

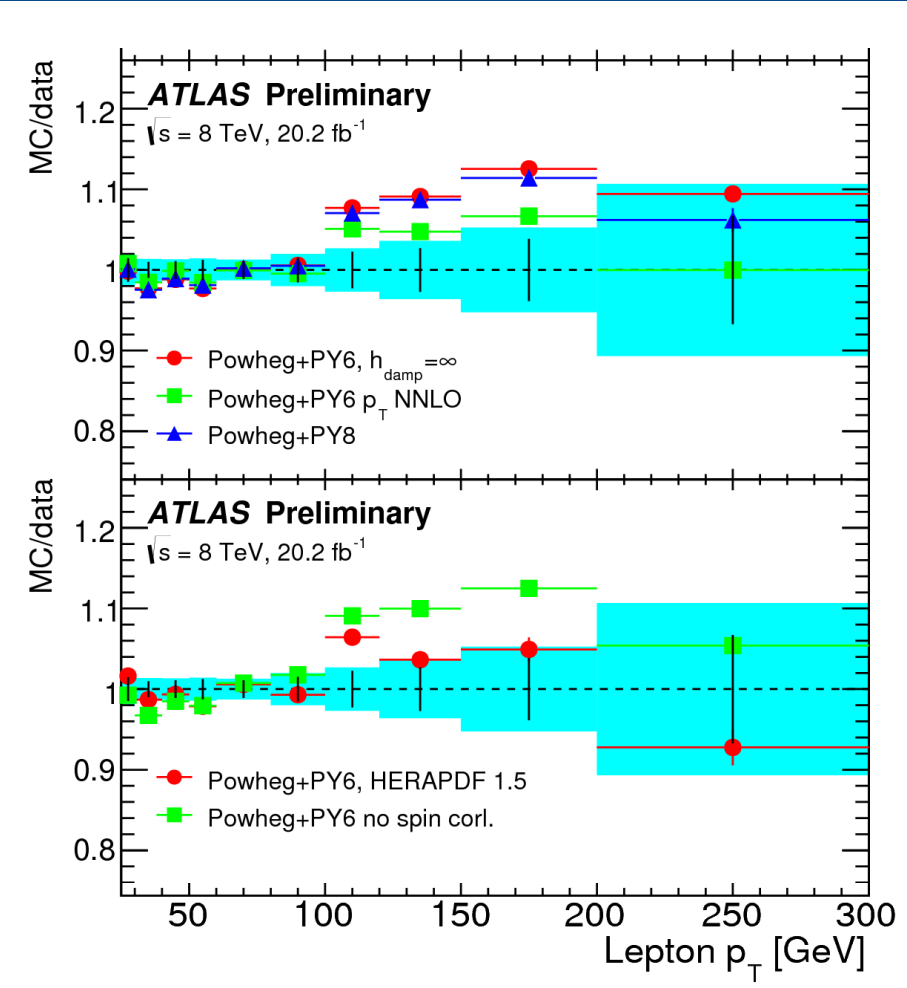

ATLAS-CONF-2017-044 b-tagging efficiency: extracted with $\sigma(\mathrm{t} \overline{\mathrm{t}})$ to reduce uncertainty

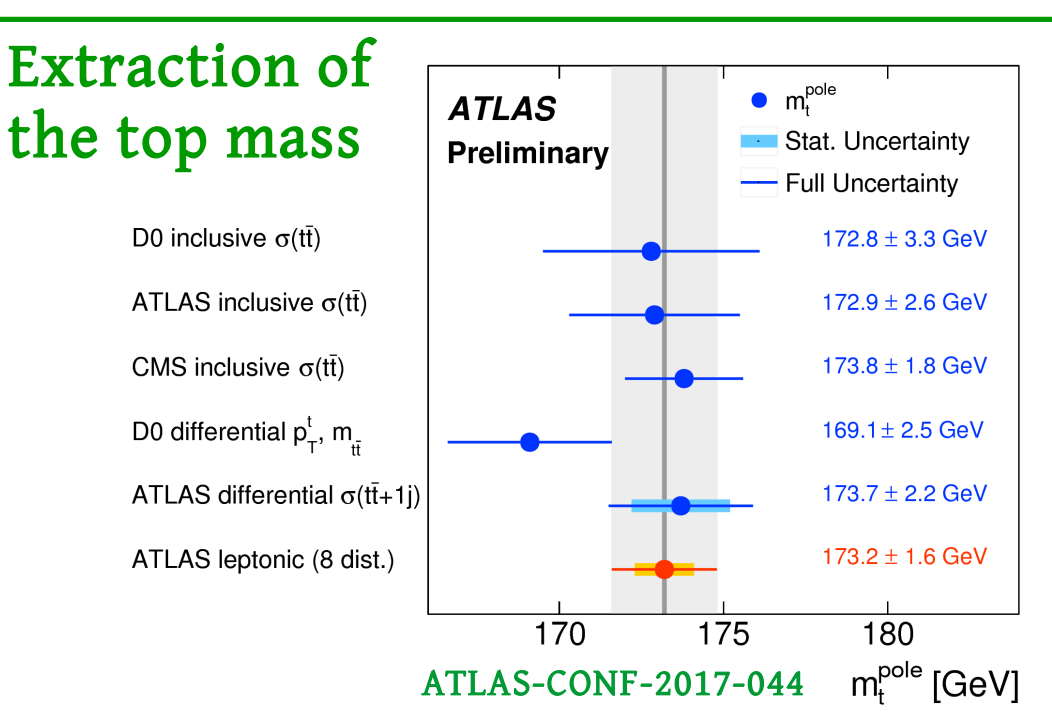

- Calculate $\chi^{2}$ with various MC NLO $m_{t}$ hypothesis, interpolation to derive $m_{t}$ at minimum

- Most precise pole mass measurement 


\section{$\mathrm{d \sigma}(\mathrm{t} \mathfrak{t})$ in e $\mu$ channel @13 TeV}

Cleanest channel for $\mathrm{t} \overline{\mathrm{t}}$ measurements but lower BR

Challenge: reconstruct the two neutrinos using $\mathrm{E}_{\mathrm{T}}$ miss

Eur. Phys. J. C77 (2016) 299
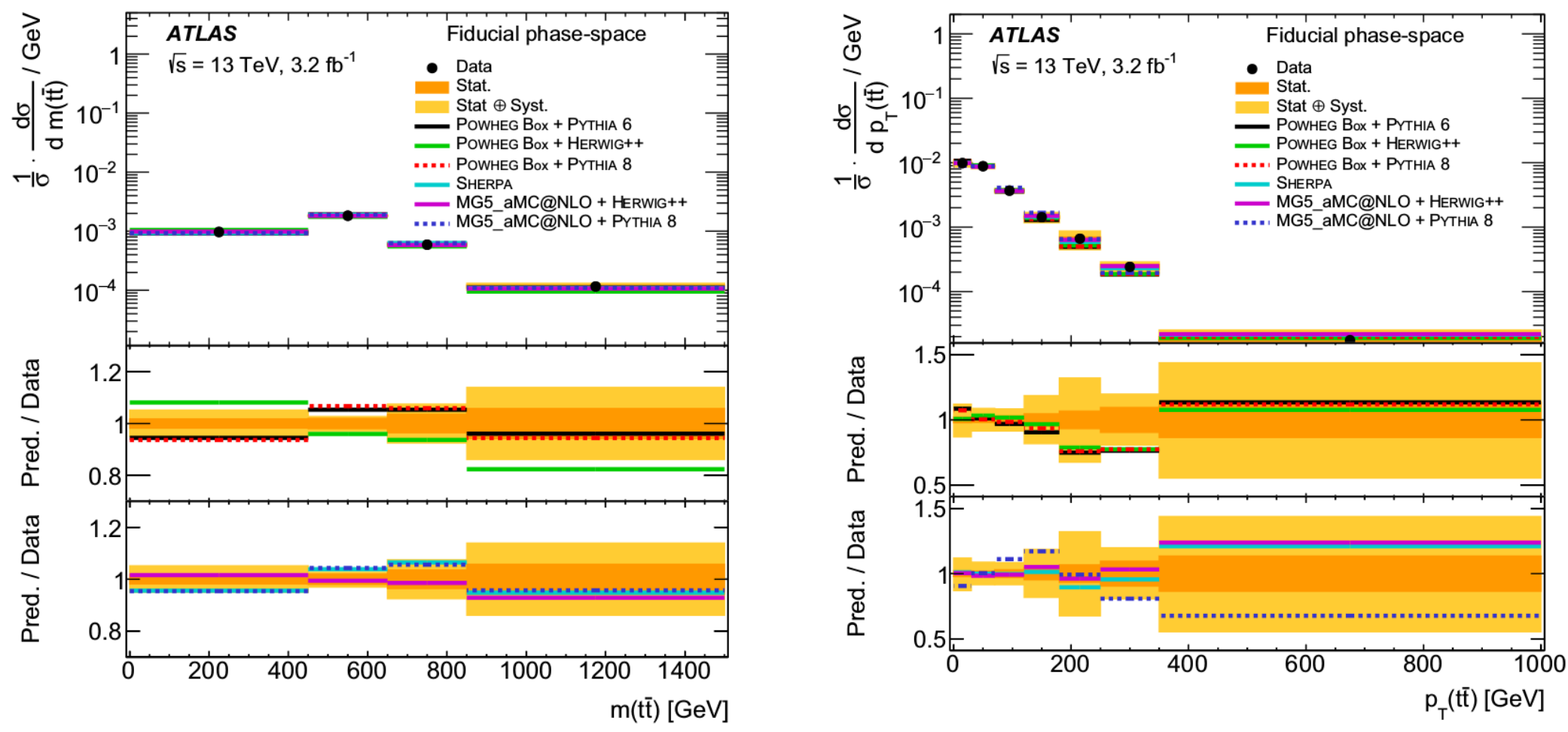

\section{Analysis strategy:}

- Required one e and one $\mu(\mathrm{OS}), \mathrm{p}_{\mathrm{T}}>25 \mathrm{GeV}$ and $2 \mathrm{~b}$-tagged jets

- Main backgrounds (MC estimate): $\mathrm{Z} / \gamma \rightarrow \tau^{+} \tau^{-}$, Diboson, Fake lepton, Single top (85\% signal purity)

- Reconstruct neutrinos from $E_{t}$ iss $u s i n g$ neutrino weighting technique, constraint on $m_{t}$ and $m_{w}$

- Unfolding to particle level

\section{Results:}

- Good agreement with SM expectations.

- Main uncertainty: top modelling 


\section{do(tt) in l+jet channel @ $13 \mathrm{TeV}$}

\section{Leptonic top:}

- Lepton

- $v: \mathrm{E}_{\mathrm{T}}^{\text {miss }}$ ( use constraint on $\mathrm{W}$ mass to find $\mathrm{p}_{\mathrm{z}}$ component)

- Closest b-jet to the lepton

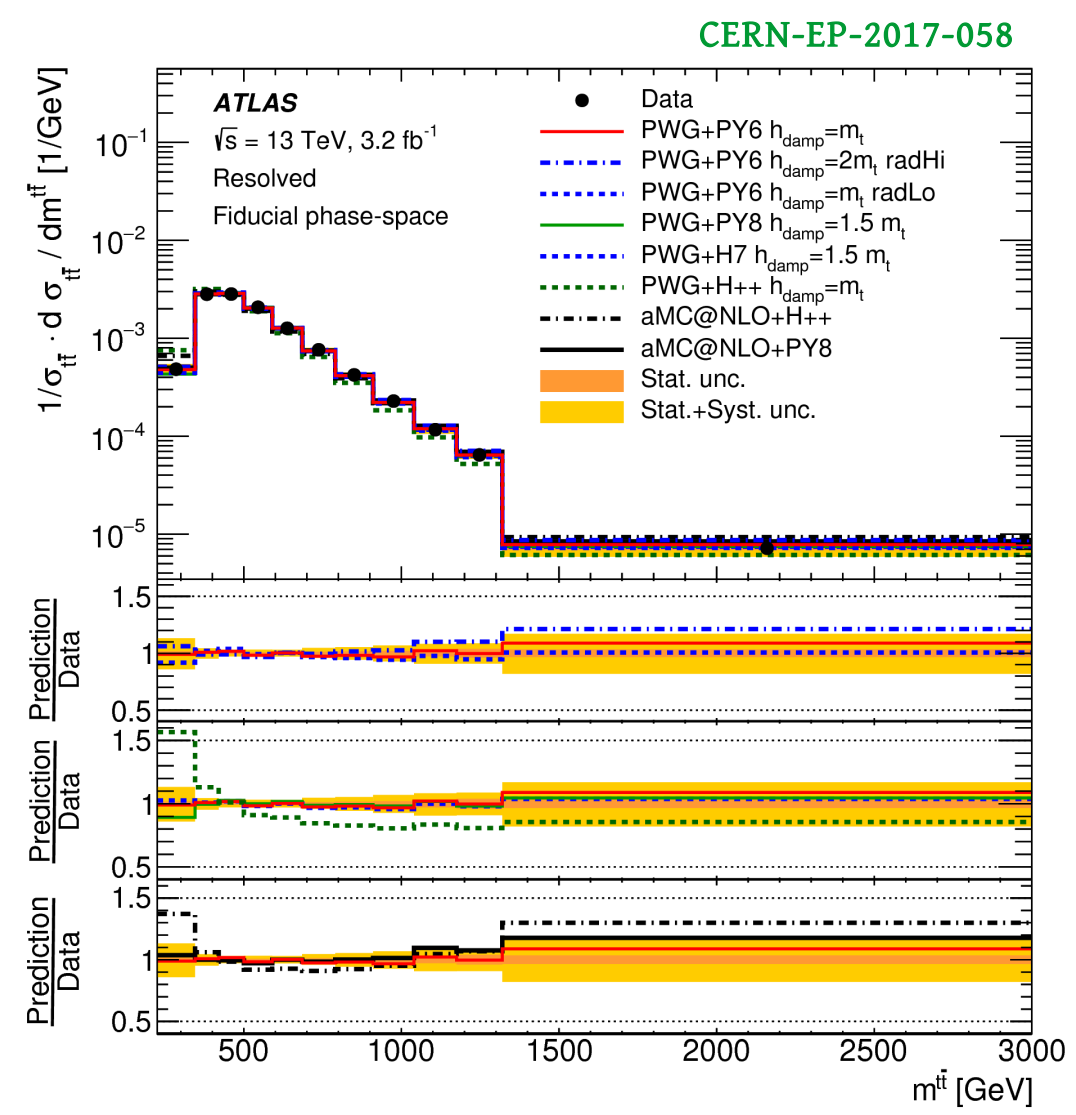

\section{Requirements:}

- Single lepton with $p_{\mathrm{T}}>25 \mathrm{GeV}$

- At least 4 jets with $p_{\mathrm{T}}>25 \mathrm{GeV}$

$\mathrm{W}$

- At least 2 bjet (if $>2$ selected the highest $\mathrm{p}_{\mathrm{T}}$ )

\section{Hadronic top:}

- Use the two light jets (invariant mass near to $\mathrm{W}$ mass)

- Other b-jet

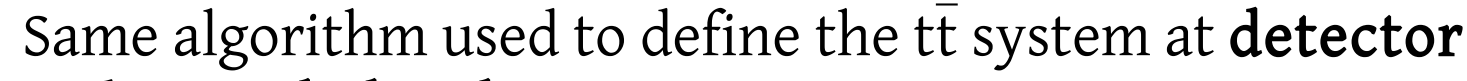
and particle level

Cut and count analysis, reaches a signal purity $89 \%$

- Dominant uncertainties: JES and b-tagging

- Good agreement with SM expectations.

- High discriminating power among various MC generator 


\section{Boosted measurements}

- High energy reached from LHC allows to explore frontier kinematic regime (TeV scale)

- If the top quark is produced with high $\mathrm{p}_{\mathrm{T}}$ the decay products have a large boost, and are contained in a:

$$
\begin{aligned}
& \Delta R=\sqrt{\Delta y+\Delta \phi} \sim \frac{2 m}{p_{T}} \\
& \left(\Delta R=1.0 \text { for }_{t}=173, p_{T}>300 \mathrm{GeV}\right)
\end{aligned}
$$

- Combine substructure properties ( $\tau_{32}$, mass) to define a tagger that select a large- $\mathrm{R}$ jet deriving from the top quark
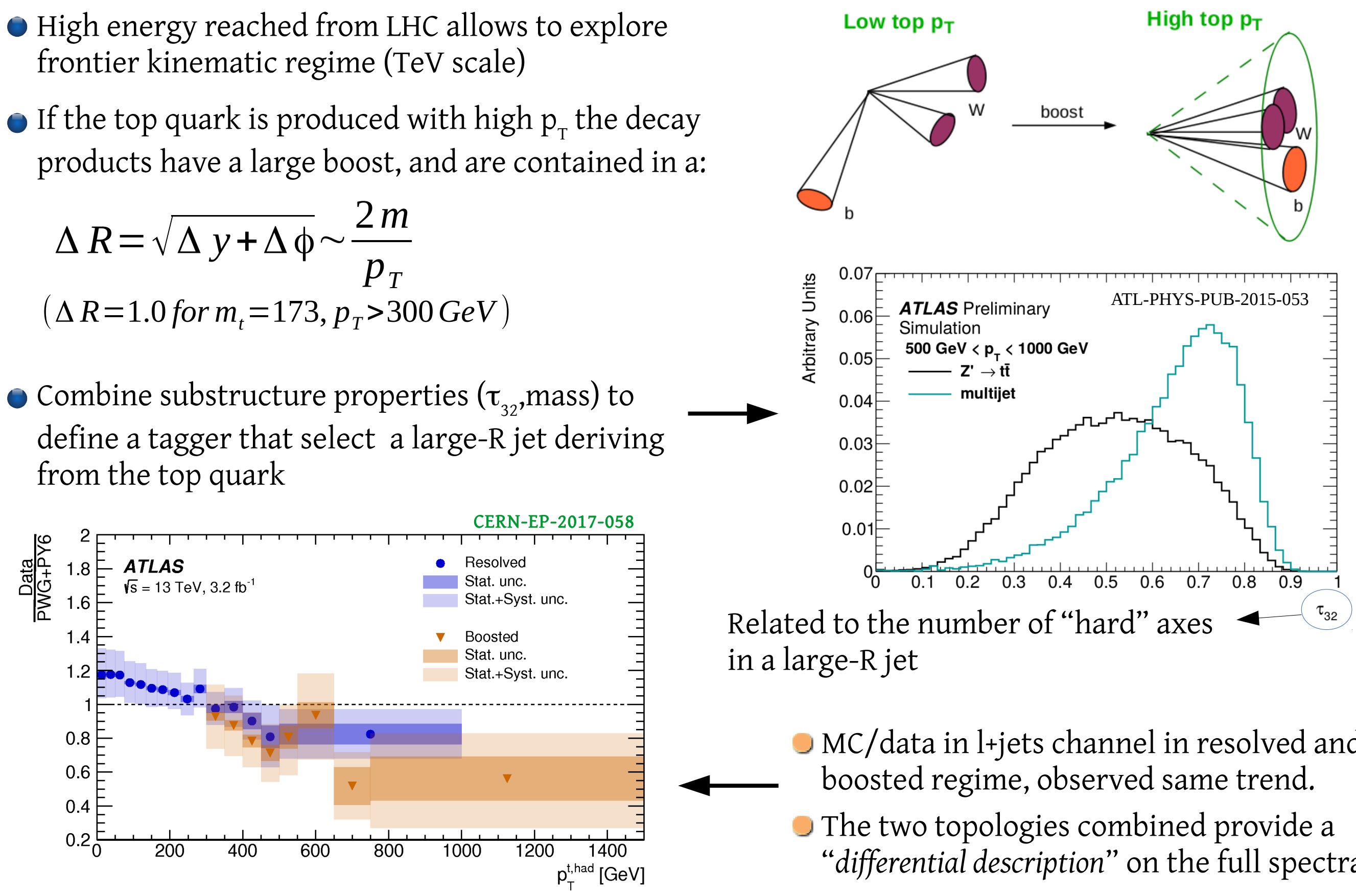


\section{Boosted measurements @ 13TeV}

\section{l+jets channel:}

Hadronic top: 1 large-R jet, $\mathrm{p}_{\mathrm{T}}>300 \mathrm{GeV}$, top tagged (80\%)

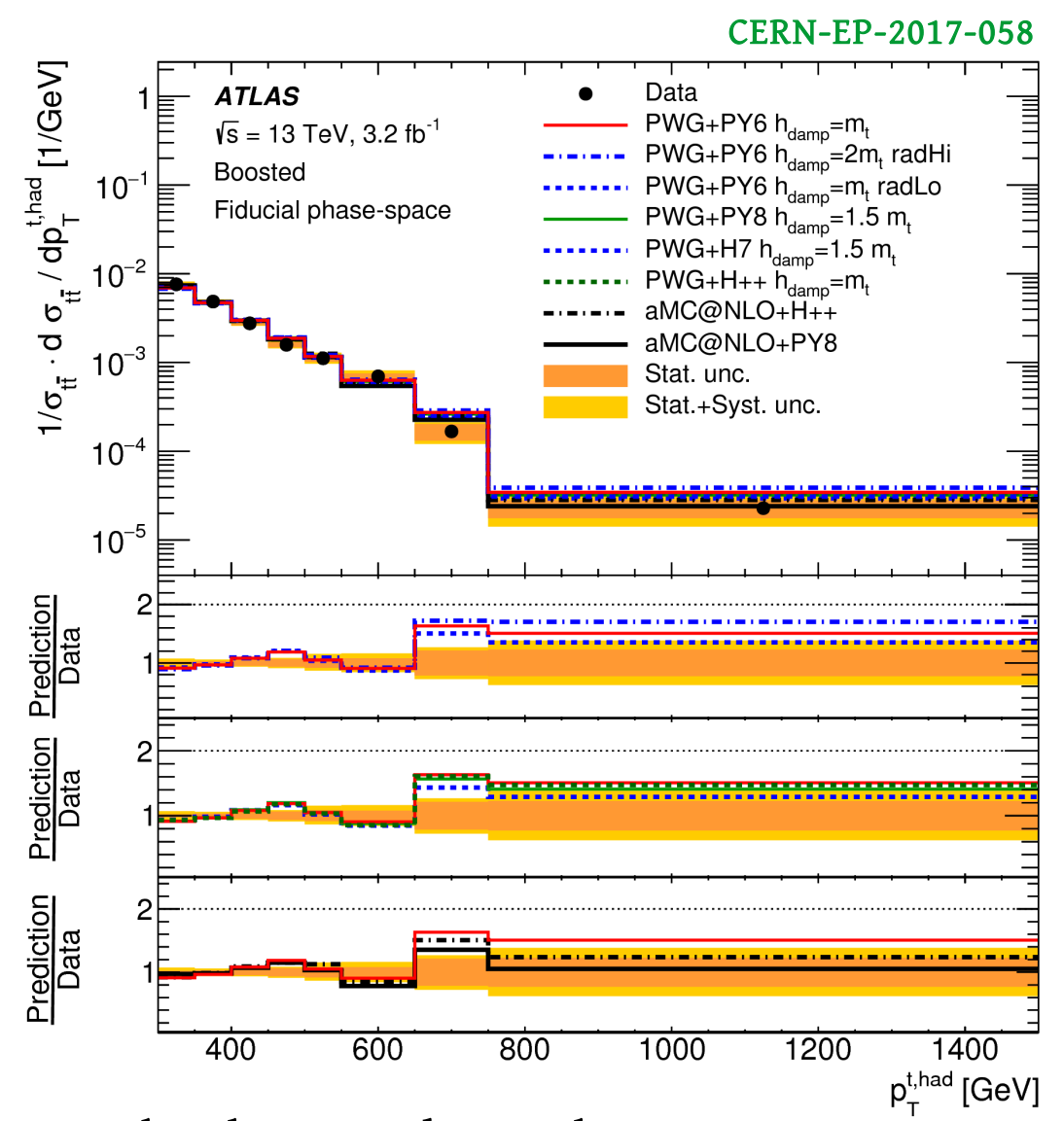

Main background: Single top, $\mathrm{W}+$ jets

- Main uncertainties: Large-R jets JES, stat

- Good agreement with SM, small tension shown at high $\mathrm{p}_{\mathrm{T}}$
All-had channel:

$\geq 2$ large-R jets top tag (50\% WP), $122.5 \mathrm{GeV}<$ $\mathrm{m}_{\text {large-R jet }}<222.5 \mathrm{GeV}$

ATLAS-CONF-2016-100
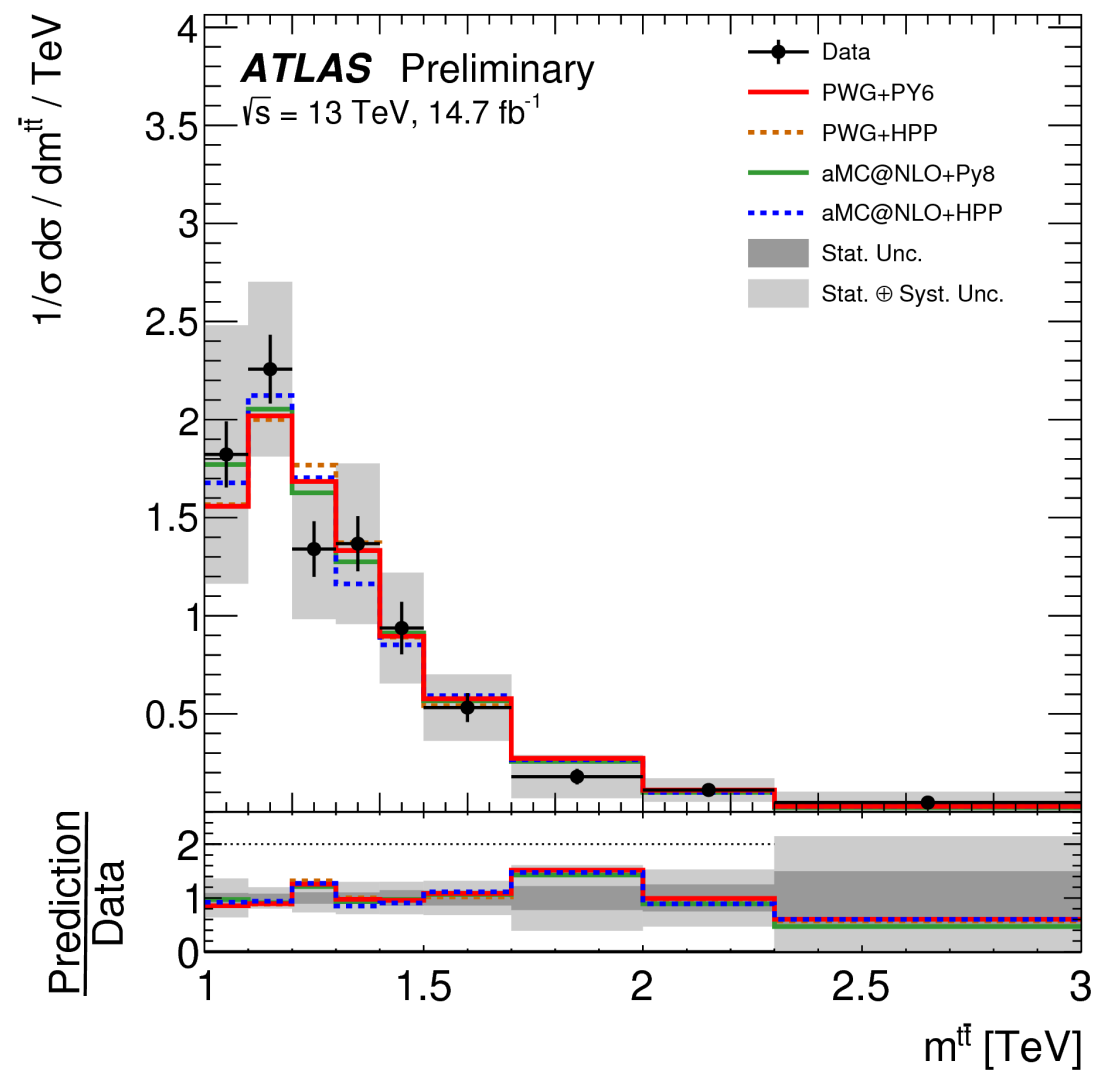

Main background: Multijet (data driven)

Main uncertainties: Large-R jets JES, stat, MC modeling.

- Good agreement with SM 


\section{Single top production}
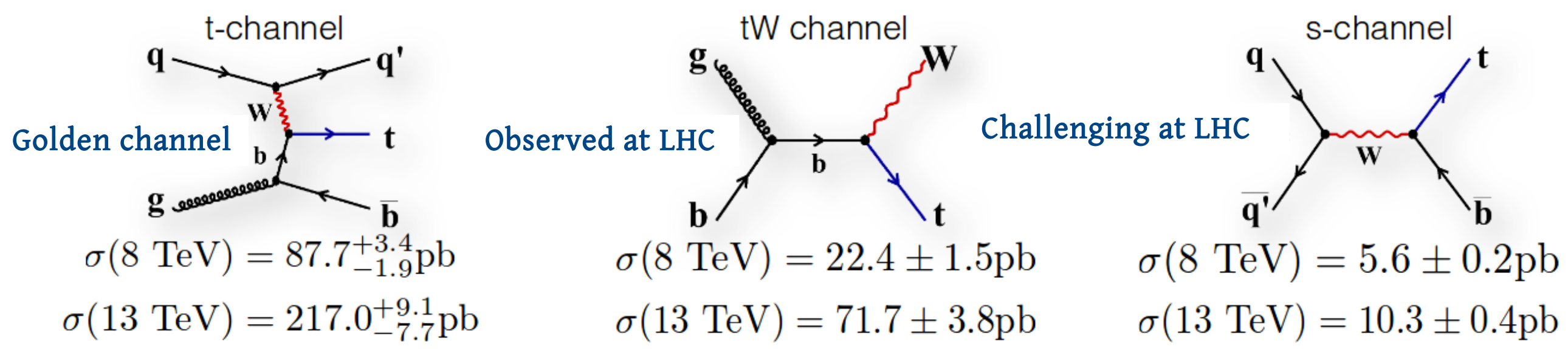

Many interesting results!! Also probing top coupling with $W$ and $Z$ bosons!

\begin{tabular}{|c|c|c|c|c|}
\hline & s-channel & $\begin{array}{c}\text { tW- } \\
\text { channel }\end{array}$ & t-channel & tZ production \\
\hline $\begin{array}{c}8 \\
\text { TeV }\end{array}$ & arXiv:1511.05980 & JHEP 01 (2016) 064 & arXiv:1702.02859 & \\
\hline $\begin{array}{c}13 \\
\text { Tev }\end{array}$ & & arXiv:1612.07231 & JHEP04(2017)086 & ATLAS-CONF-2017-052 \\
\hline
\end{tabular}

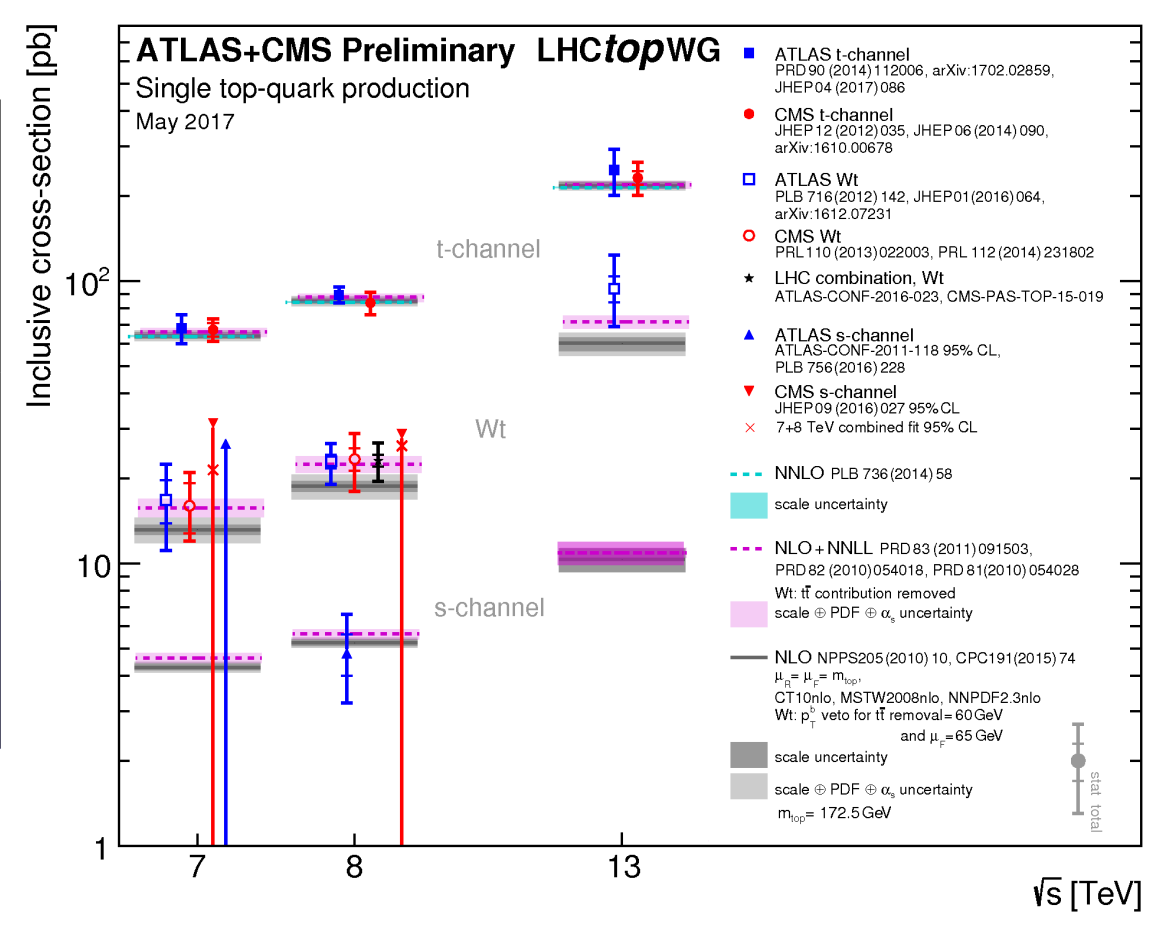




\section{do(t), t-channel @8TeV using NN}

- Channel with highest production $\sigma$

- The top production is favored by PDF with respect to the anti-top production

\section{Analysis strategy:}
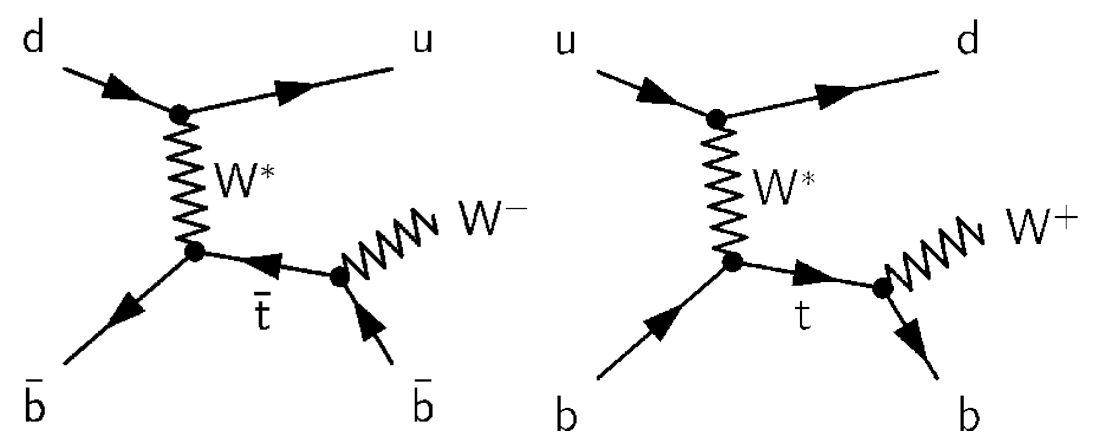

- Discriminate signal and background using a NN based on:

$\rightarrow \mathrm{m}(\mathrm{lvb}), \mathrm{m}(\mathrm{bj})$ and the $(\mathrm{b}, \mathrm{j})$ system

$\rightarrow$ Kinematic and angular properties of $1, \mathrm{j}, \mathrm{b}, \mathrm{E}^{\mathrm{T}}{ }_{\text {miss }}$

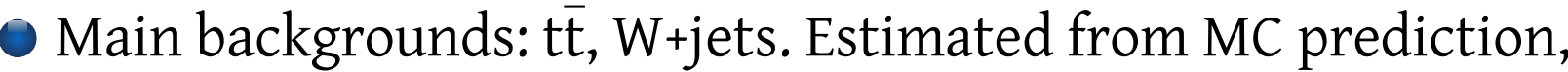
constrained with a likelihood fit on NN output variable $\left(\mathrm{O}_{\mathrm{NN}}\right)$

- Data-driven QCD background estimate
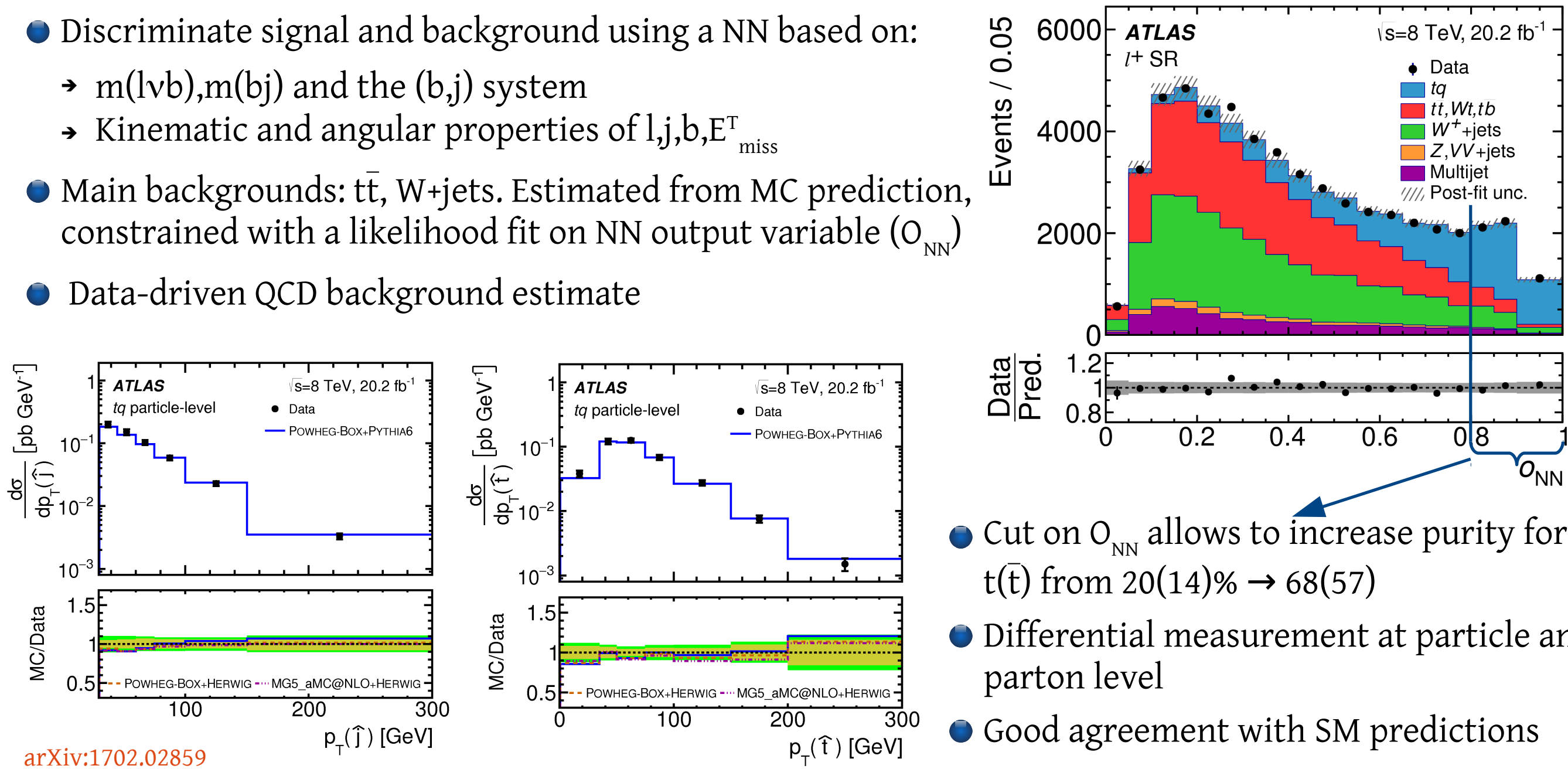

- Cut on $\mathrm{O}_{\mathrm{NN}}$ allows to increase purity for $t(\bar{t})$ from $20(14) \% \rightarrow 68(57)$

- Differential measurement at particle and parton level

- Good agreement with SM predictions 


\section{$\sigma(t), t$-channel @ 8,13 TeV}

Same NN technique applied to extract inclusive cross section but applying a likelihood fit using full $\mathrm{NN}$

The total cross section measurement allows the extraction of the element of $C K M\left|V_{t b}\right|$

- Technique similar to $8 \mathrm{TeV}$ applied to $13 \mathrm{TeV}$, slightly different $\mathrm{NN}$

- Measurement of $\mathrm{R}_{\mathrm{t}}=\sigma(\mathrm{t}) / \sigma(\mathrm{t})$ highly sensitive to PDFs

\section{$8 \mathrm{TeV}$}

$13 \mathrm{TeV}$ JHEP04(2017)086

$$
\begin{gathered}
\sigma_{\text {tot }}(t q)=56.7_{-3.8}^{+4.3} \mathrm{pb} \quad \sigma_{\text {tot }}(\bar{t} q)=32.9_{-2.7}^{+3.0} \mathrm{pb} \\
\sigma_{\text {tot }}(t q+\bar{t} q)=89.6_{-6.3}^{+7.1} \mathrm{pb} \\
f_{\mathrm{LV}} \cdot\left|V_{t b}\right|=1.029 \pm 0.048
\end{gathered}
$$

Main unc:: JES, top modelling

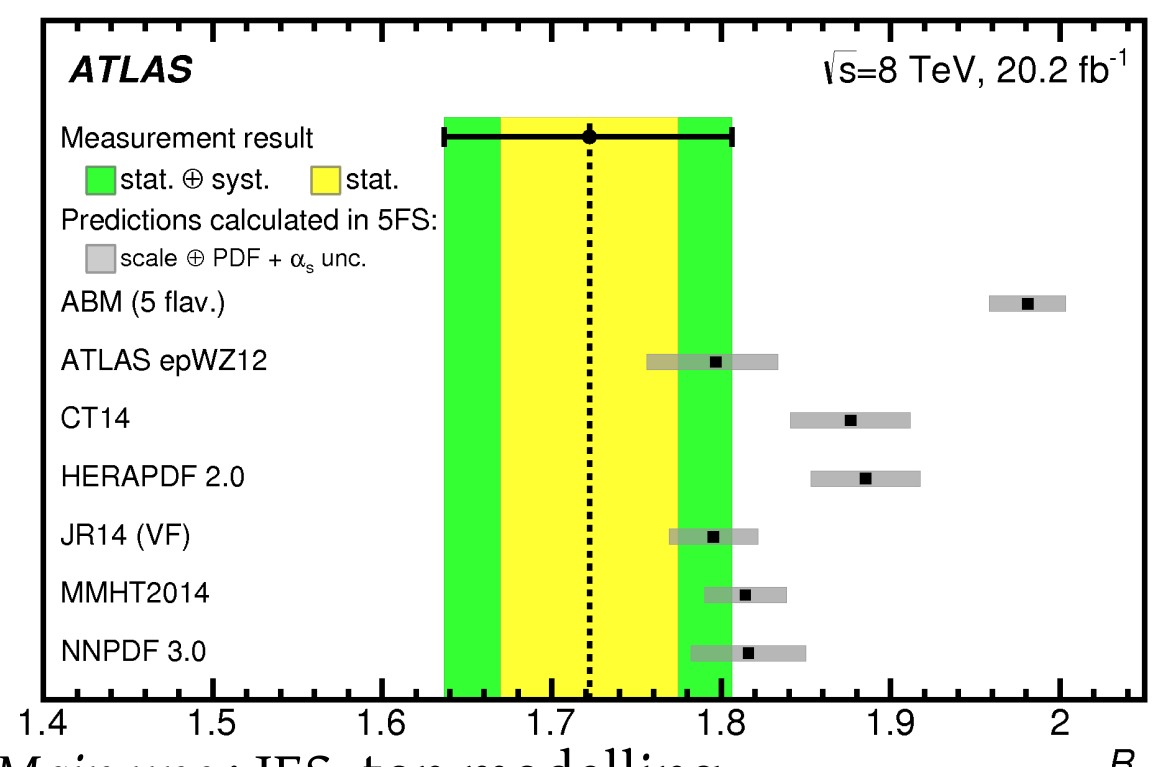

Main unc:: JES, top modelling

$$
\begin{aligned}
& \sigma(t q)=156 \pm 5 \text { (stat.) } \pm 27 \text { (syst.) } \pm 3 \text { (lumi.) pb } \\
& \sigma(\bar{t} q)=91 \pm 4 \text { (stat.) } \pm 18 \text { (syst.) } \pm 2 \text { (lumi.) pb } \\
& \sigma(t q+\bar{t} q)=247 \pm 46 \mathrm{pb} . \\
& f_{\mathrm{LV}} \cdot\left|V_{t b}\right|=1.07 \pm 0.09 .
\end{aligned}
$$

Main unc.: JES, b-tagging, top modelling

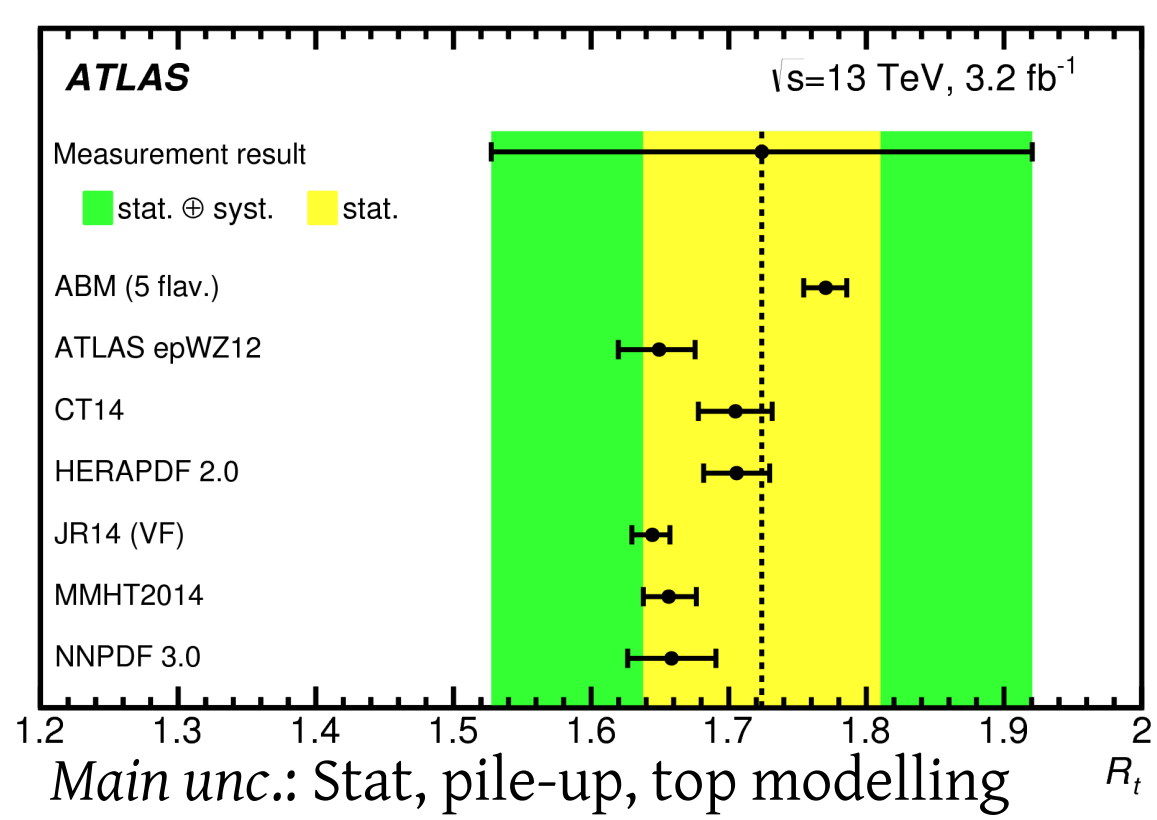




\section{$\sigma(t)$ in Wt-channel @ 13TeV using BDT}

Second leading process in single top production.

- Not observable at Tevatron, production observed for the first time at LHC @8 TeV

\section{Analysis Strategy:}

- Event selection aims to detect $\mathrm{Wt}$ and $\mathrm{t} \overline{\mathrm{t}}$ events, highly reducing $\mathrm{Z}+\mathrm{jet}$ background ( $\mathrm{m}_{l l}$ and $\mathrm{E}_{\mathrm{T}}{ }^{\text {miss }}$ cuts)

- All backgrounds estimated by simulation

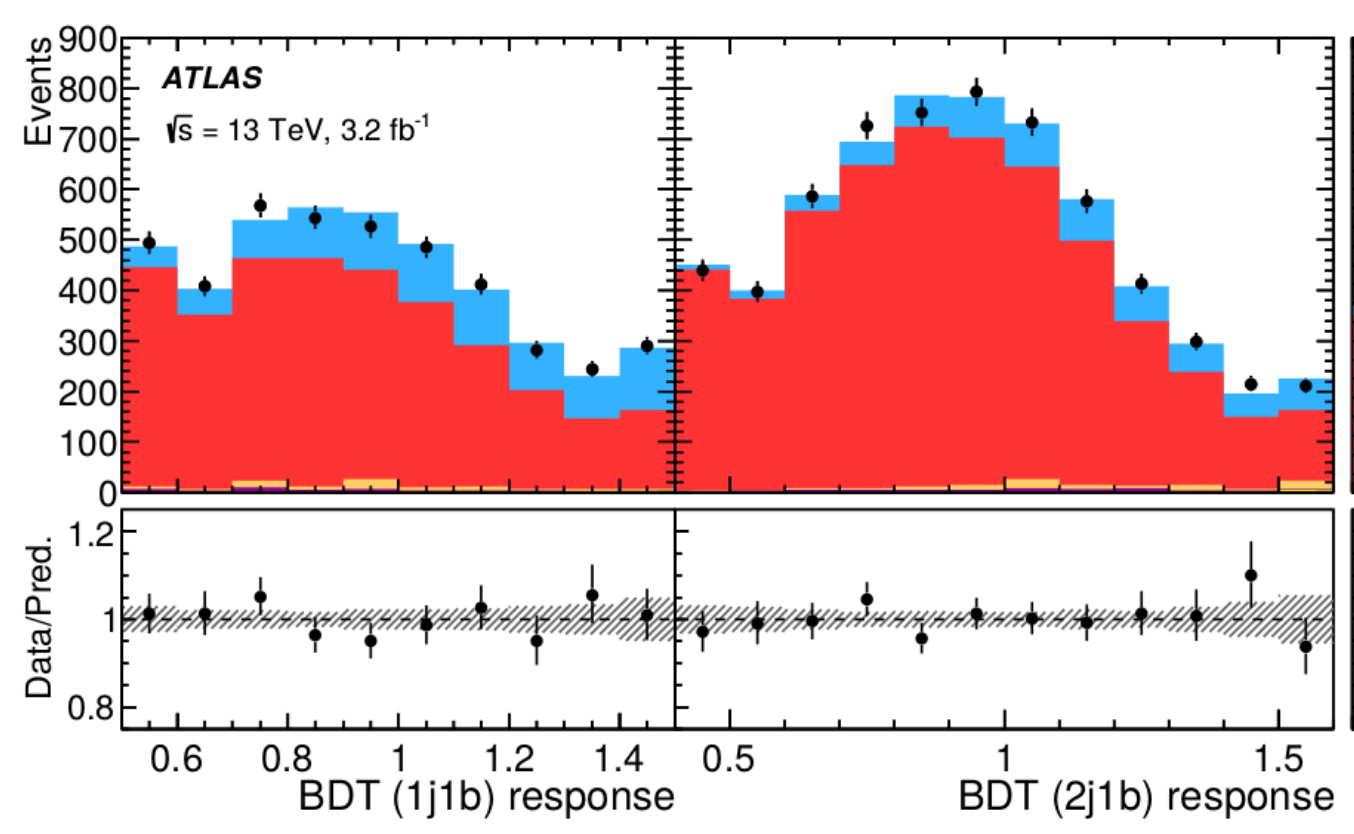

$\sigma_{W t}=94 \pm 10$ (stat.) ${ }_{-22}^{+28}$ (syst.) \pm 2 (lumi.) pb.

Significance: $4.5 \sigma(3.9 \sigma)$

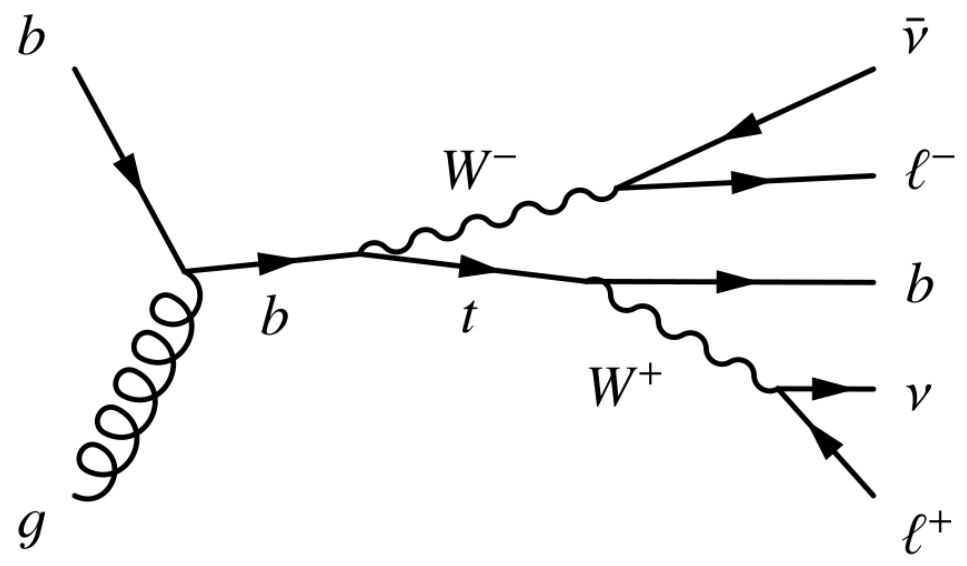

arXiv:1612.07231

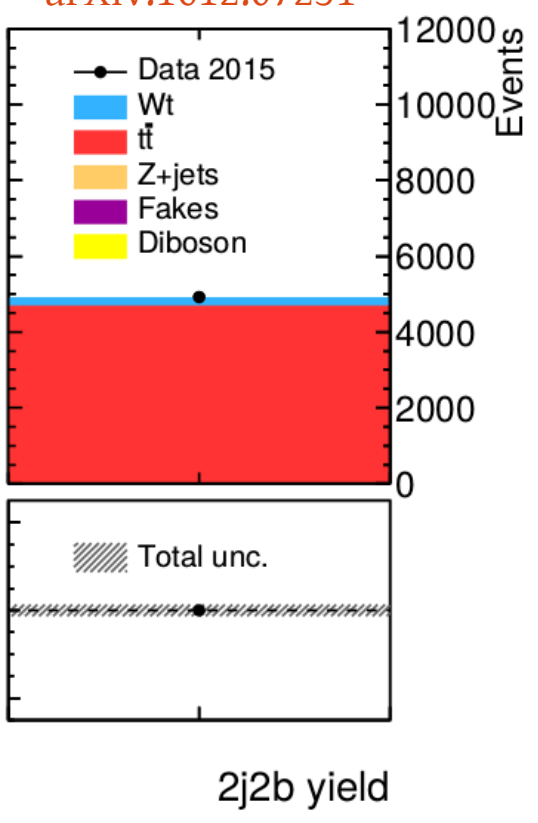

Main uncertainty: jet energy resolution, top modelling and jet energy scale 


\section{$\sigma(t Z) @ 13 \mathrm{TeV}$, using NN}

Electroweak process no yet observed, very small production $\sigma$ Sensitive to $t Z$ and WWZ coupling

\section{Analysis strategy:}

- Trilepton channel most pure for first observation, despite small BR (2.2\%)

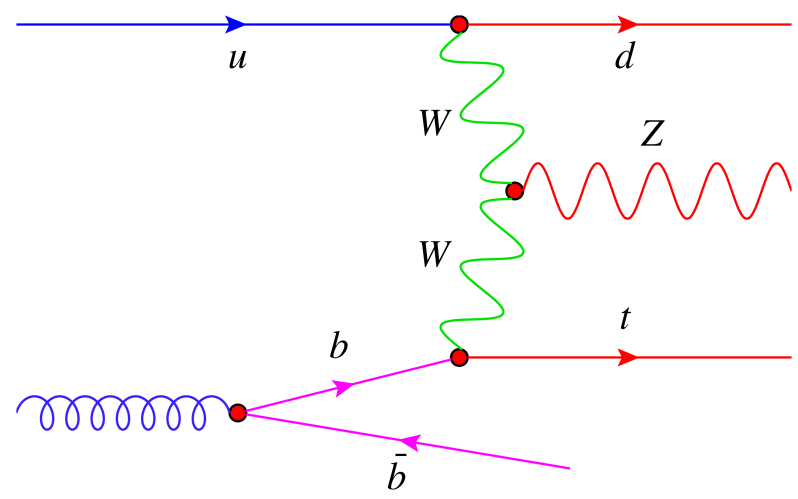

- Main background: Diboson (WZ), Z+jets events with mis-identified lepton (data driven), t $\overline{\mathrm{t}}$ (data driven normalization), $\mathrm{t} \overline{\mathrm{t}} \mathrm{V}$ (MC estimated)

- After pre-selection events are divided into SR(2j1b), and $\mathrm{VR}, \mathrm{CR}$ to check the main background description and normalization

- NN is built to improve signal to the background separation, binned likelihood fit in SR used to extract $\sigma(t Z q)$

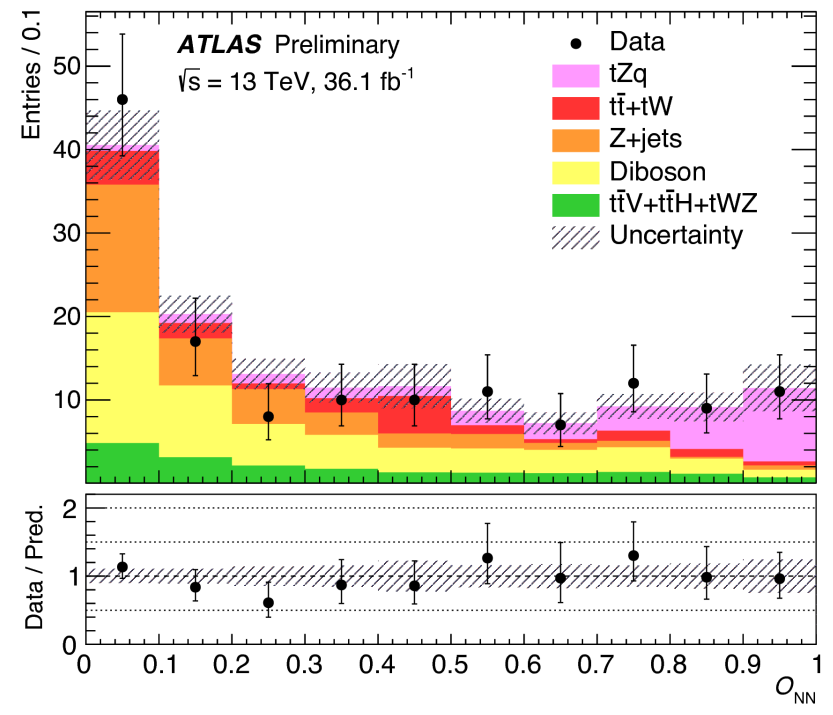

$$
\begin{gathered}
\sigma_{t Z q}=600 \pm 170 \text { (stat.) } \pm 140 \text { (syst.) fb } \\
\sigma_{t z q}^{\text {theor }}=800+48.8 \mathrm{fb} \quad \text { Main uncertainty: } \\
-59.2
\end{gathered}
$$

Main uncertainty: $t Z q$ modelling, jet

Significance: $4.2 \sigma(5.4 \sigma)$

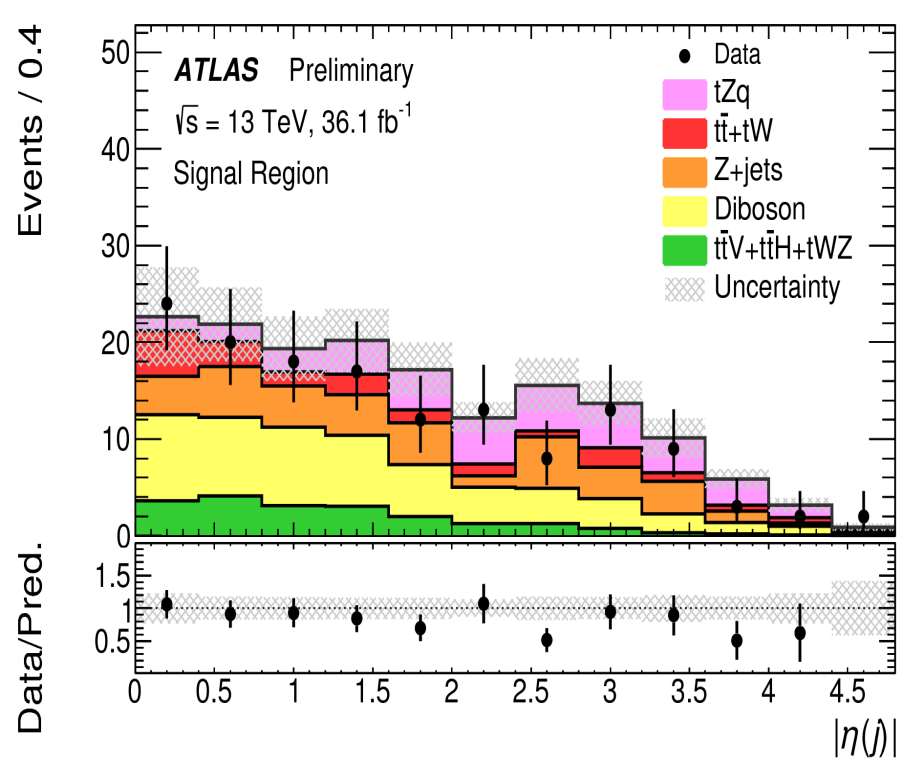




\section{Summary}

ATLAS provided a huge number of measurements for the $t$ and $t \bar{t}$ production at 8 and $13 \mathrm{TeV}$.

\section{Measurement of $t \bar{t}$ :}

- Inclusive and differential measurements show a good agreement with the SM expectations.

- Used as input for MC tuning and PDF fitting, a possible application is also the pole mass extraction.

- Used to set limits to the existence of new physics.

- Boosted techniques can be employed to extract the cross section also in region where the top has a very high $\mathrm{p}_{\mathrm{T}}$.

\section{Single top measurement:}

- Presented the latest measurement in $\mathrm{t}$-channel, and $\mathrm{Wt}$ channel.

- Observed a general good agreement with the SM expectations.

- The measurements can be used to improve PDF extraction and extract the $\left|\mathrm{V}_{\mathrm{tb}}\right|$.

- Presented the first measurement of $\sigma(t z)$ (observed with a $4.2 \sigma$ significance).

All the measurements will be tuned and improved with the incoming data that will allow to reduce uncertainty and increase the events produced in challenging channels and regimes.

\section{Thank you for your attention!!!}




\section{Back Up}




\section{Multivariate approach}

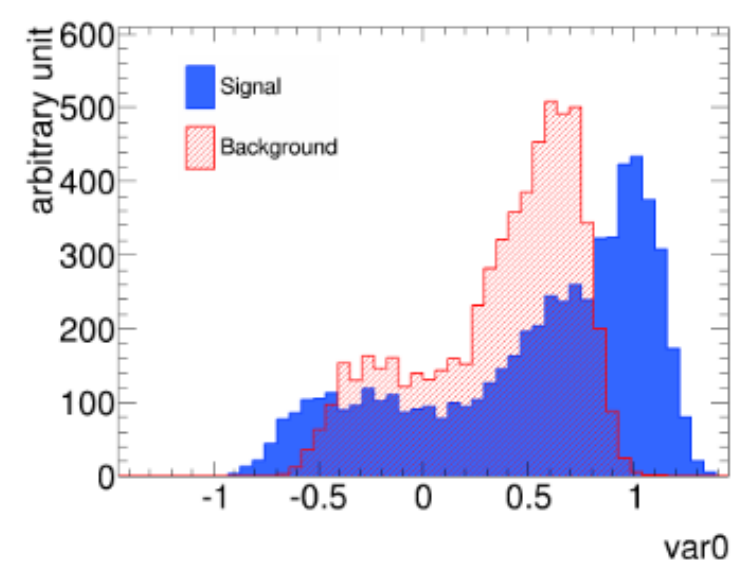

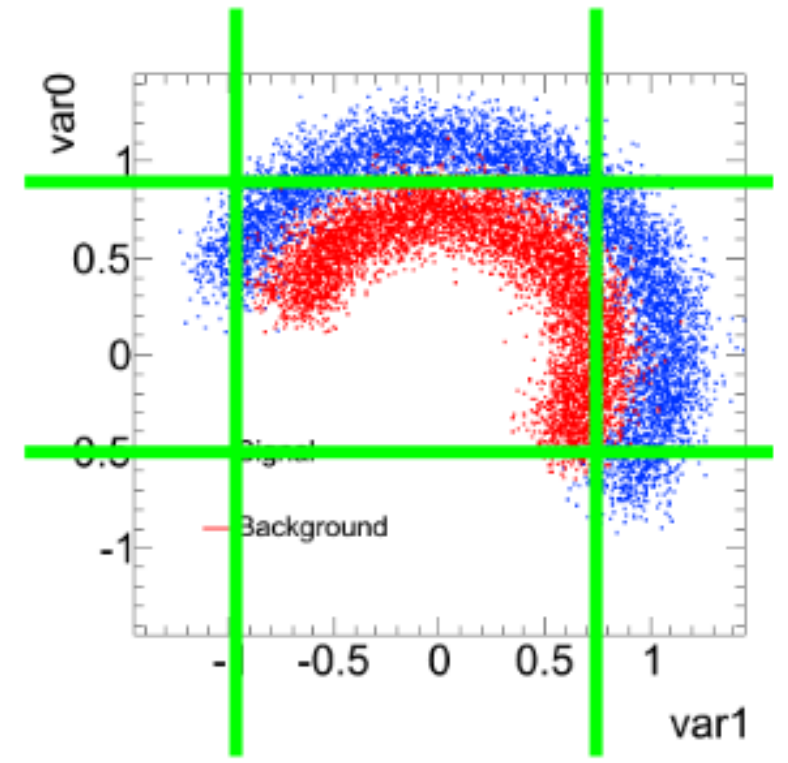

Take into account correlation
Various approach to combine the informations

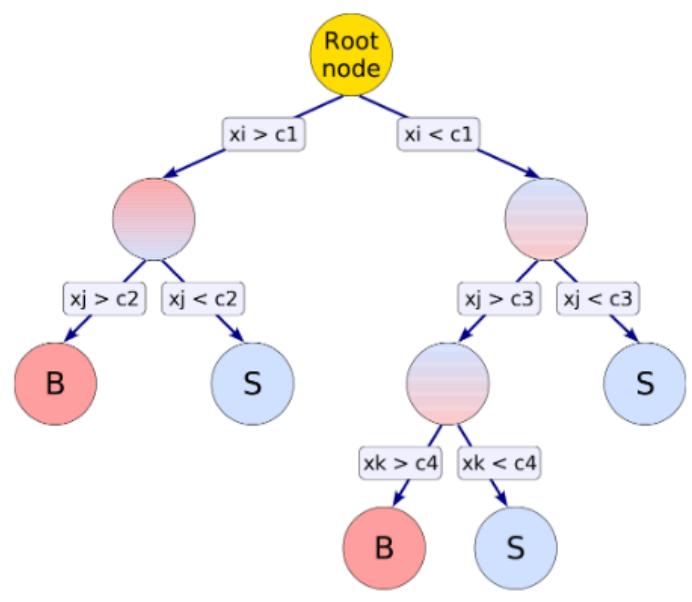

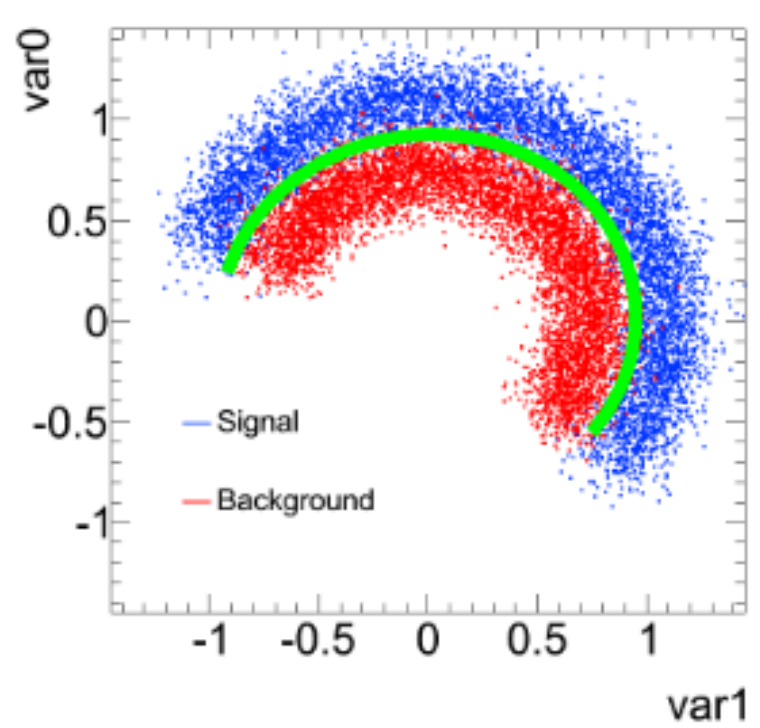

Combine in a smart way the variables, to get higher separation

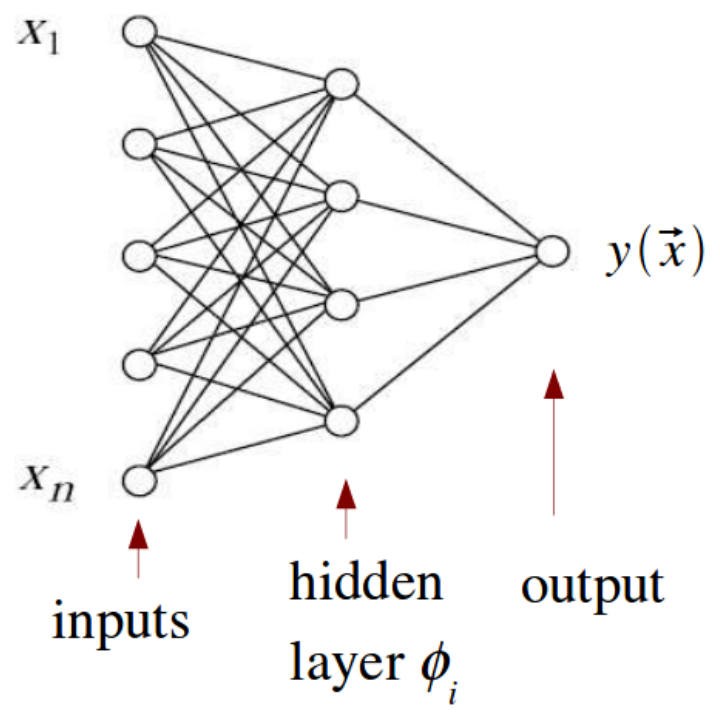




\section{Measurement of $\sigma(\mathrm{tt})$ in $\tau+j e t s @ 8 \mathrm{TeV}$}

- Good cross check to the cross section measurement performed in other channel

- BSM mass and flavour dependent couplings are expected to enhance the $t \rightarrow \tau v_{\tau} b$ cross section (SUSY, 2HDM includes $\mathrm{t} \rightarrow \mathrm{Hq} \rightarrow \tau \tau q$ )

\section{Event selection:}

Reconstructed employing a BDT, optimized to identify:
proton




\section{$\sigma(t \mathfrak{t})$ in all-had channel @13 TeV}

\section{Event selection:}

- no e $/ \mu p_{\mathrm{T}}>25 \mathrm{GeV}$

- 2 large-R jets with $\mathrm{p}_{\mathrm{T}}>350 \mathrm{GeV}$ and $|\eta|<2.0$

- leading large-R jet $\mathrm{p}_{\mathrm{T}}>500 \mathrm{GeV}$

- A small-R (0.4) jet with $\mathrm{p}_{\mathrm{T}}>25 \mathrm{GeV}$ and $|\eta|<2.5$

- The two highest $\mathrm{p}_{\mathrm{T}}$ large-R jets need to be top tagged (50\% WP) and satisfy $122.5 \mathrm{GeV}<\mathrm{m}_{\mathrm{J}}<222.5$ $\mathrm{GeV}$

- The two large-R jets need to be matched (DR $<1.0)$ with a b-tagged small-R jet.

\begin{tabular}{lrlr}
\hline$t \bar{t}$ (all-hadronic) & 1190 & \pm & 240 \\
\hline$t \bar{t}$ (non all-hadronic) & 60 & \pm & 15 \\
Single top-quark & 9 & \pm & 5 \\
Multijet events & $\mathrm{s} 300$ & \pm & 20 \\
\hline Prediction & 1570 & \pm & 260 \\
Data $\left(14.7 \mathrm{fb}^{-1}\right)$ & 1512 & & \\
\hline
\end{tabular}




\section{$\mathrm{d \sigma}(\mathrm{t} \overline{\mathrm{t}})$ in $\mathrm{e} / \mu$ channel @ $8 \mathrm{TeV}$}

\begin{tabular}{|c|c|c|}
\hline Event counts & $N_{1}$ & $\mathrm{~N}_{2}$ \\
\hline Data & 21666 & 11739 \\
\hline$W t$ single top & $2080 \pm 210$ & $350 \pm 120$ \\
\hline$Z(\rightarrow \tau \tau \rightarrow e \mu)+$ jets & $210 \pm 40$ & $7 \pm 2$ \\
\hline Diboson Data drive & n $120 \pm 30$ & $3 \pm 1$ \\
\hline Misidentified leptons & $220 \pm 80$ & $78 \pm 50$ \\
\hline Total background & $2630 \pm 230$ & $440 \pm 130$ \\
\hline
\end{tabular}

ATLAS-CONF-2017-044

Effect of the measurement of $\left|\eta^{\text {lep. }}\right|,\left|y^{\text {eu }}\right|$, and $E^{\mathrm{e}}+E^{\mu}$ in $t \bar{t}$ events on PDF fitting in HERAPDF:
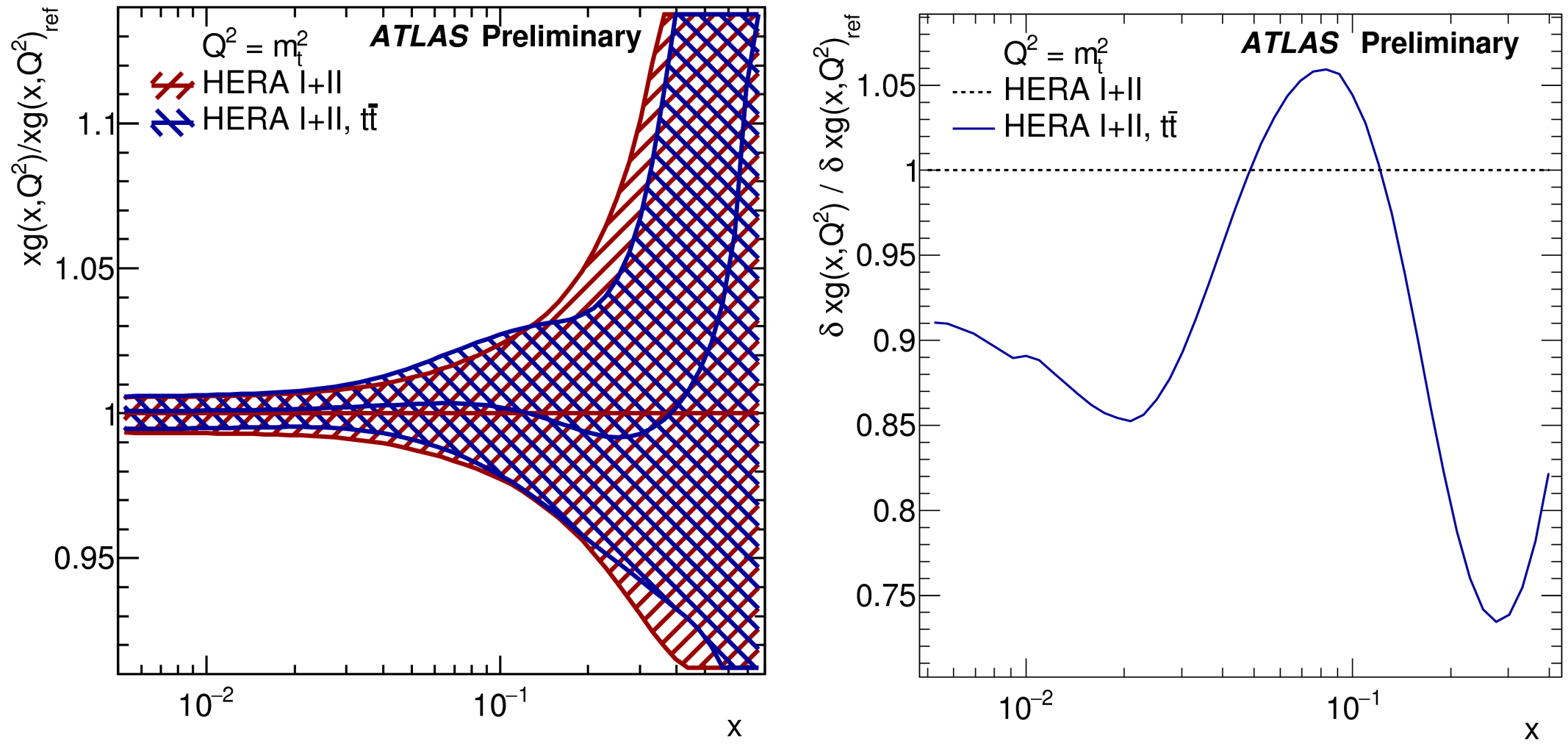


\section{t-channel: Rt uncertainty}

\section{$8 \mathrm{TeV}$}

\begin{tabular}{lc}
\hline Source & $\Delta R_{t} / R_{t}[\%]$ \\
\hline Data statistics & \pm 3.0 \\
Monte Carlo statistics & \pm 1.8 \\
\hline Background modelling & \pm 0.7 \\
Jet reconstruction & \pm 0.5 \\
$E_{\mathrm{T}}^{\text {miss modelling }}$ & \pm 0.6 \\
$t q(\bar{t} q)$ NLO matcling & \pm 0.5 \\
$t q(\bar{t} q)$ scale variations & \pm 0.7 \\
$t \bar{t}$ NLO matching & \pm 2.3 \\
$t \bar{t}$ parton shower & \pm 1.7 \\
PDF & \pm 0.7 \\
\hline Total systematic & \pm 3.9 \\
Total (stat. + syst.) & \pm 5.0 \\
\hline
\end{tabular}

\section{$13 \mathrm{TeV}$}

\begin{tabular}{lccc}
\hline Source & $\frac{\Delta \sigma(t q)}{\sigma(t q)}[\%]$ & $\frac{\Delta \sigma(\bar{t} q)}{\sigma(\bar{t} q)}[\%]$ & $\frac{\Delta R_{t}}{R_{t}}[\%]$ \\
\hline Data statistics & \pm 2.9 & \pm 4.1 & \pm 5.0 \\
Monte Carlo statistics & \pm 2.8 & \pm 4.2 & \pm 5.1 \\
\hline
\end{tabular}

\section{Reconstruction efficiency and calibration uncertainties}

Muon uncertainties

Electron uncertainties

$\pm 0.8$

$<0.5$

$\pm 0.9$

$\pm 1.0$

JES

Jet energy resolution

$\pm 3.4$

$E_{\mathrm{T}}^{\mathrm{miss}}$ modelling

$\pm 3.9$

$b$-tagging efficiency

$\pm 0.9$

$c$-tagging efficiency

Light-jet tagging efficiency

$\pm 7.0$

$<0.5$

$<0.5$

$\pm 1.5$

Monte Carlo generators

$t q$ parton shower generator
$t q$ NLO matching
$t q$ radiation
$t \bar{t}, W t, t \bar{b}+\bar{t} b$ parton shower generator
$t \bar{t}, W t, t \bar{b}+\bar{t} b$ NLO matching
$t \bar{t}, W t, t \bar{b}+\bar{t} b$ radiation

$\pm 13.0$

$\pm 2.1$

$\pm 3.7$

$\pm 3.2$

$\pm 4.4$

$<0.5$

$\pm 0.6$

Background normalisation

\begin{tabular}{lccc} 
Multijet normalisation & \pm 0.3 & \pm 2.0 & \pm 1.8 \\
Other background normalisation & \pm 0.4 & \pm 0.5 & $<0.5$ \\
Luminosity & \pm 2.1 & \pm 2.1 & $<0.5$ \\
\hline Total systematic uncertainty & \pm 17.5 & \pm 20.0 & \pm 10.2 \\
Total uncertainty & \pm 17.8 & \pm 20.4 & \pm 11.4 \\
\hline
\end{tabular}




\section{t-channel: NN comparison}

\section{$8 \mathrm{TeV}$}

\begin{tabular}{ll}
\hline Variable symbol & Definition \\
\hline$m(j b)$ & The invariant mass of the untagged jet $(j)$ and the $b$-tagged jet $(b)$. \\
$|\eta(j)|$ & The absolute value of the pseudorapidity of the untagged jet. \\
$m(\ell \nu b)$ & The invariant mass of the reconstructed top quark. \\
$m_{\mathrm{T}}\left(\ell E_{\mathrm{T}}^{\mathrm{miss}}\right)$ & The transverse mass of the lepton- $E_{\mathrm{T}}^{\text {miss }}$ system, as defined in Eq. $(? ?)$. \\
$|\Delta \eta(\ell \nu, b)|$ & The absolute value of $\Delta \eta$ between the reconstructed $W$ boson and the $b$-tagged jet. \\
$m(\ell b)$ & The invariant mass of the charged lepton $(\ell)$ and the $b$-tagged jet. \\
$\cos \theta^{*}(\ell, j)$ & The cosine of the angle, $\theta^{*}$, between the charged lepton and the untagged \\
& jet in the rest frame of the reconstructed top quark. \\
\hline
\end{tabular}

\section{$13 \mathrm{TeV}$}

\begin{tabular}{|c|c|}
\hline Variable & Definition \\
\hline$m(\ell \nu b)$ & $\begin{array}{l}\text { top-quark mass reconstructed from the charged lepton, } \\
\text { neutrino, and } b \text {-tagged jet }\end{array}$ \\
\hline$m(j b)$ & invariant mass of the $b$-tagged and untagged jet \\
\hline$m_{\mathrm{T}}\left(\ell E_{\mathrm{T}}^{\mathrm{miss}}\right)$ & transverse mass of the reconstructed $W$ boson \\
\hline$|\eta(j)|$ & modulus of the pseudorapidity of the untagged jet \\
\hline$m(\ell b)$ & invariant mass of the charged lepton $(\ell)$ and the $b$-tagged jet \\
\hline$\eta(\ell \nu)$ & rapidity of the reconstructed $W$ boson \\
\hline$\Delta R(\ell \nu b, j)$ & $\Delta R$ of the reconstructed top quark and the untagged jet \\
\hline $\cos \theta^{*}(\ell, j)$ & $\begin{array}{l}\text { cosine of the angle } \theta^{*} \text { between the charged lepton and the untagged jet } \\
\text { in the rest frame of the reconstructed top quark }\end{array}$ \\
\hline$\Delta p_{\mathrm{T}}(\ell \nu b, j)$ & $\Delta p_{\mathrm{T}}$ of the reconstructed top quark and the untagged jet \\
\hline$\Delta R(\ell, j)$ & $\Delta R$ of the charged lepton and the untagged jet \\
\hline
\end{tabular}
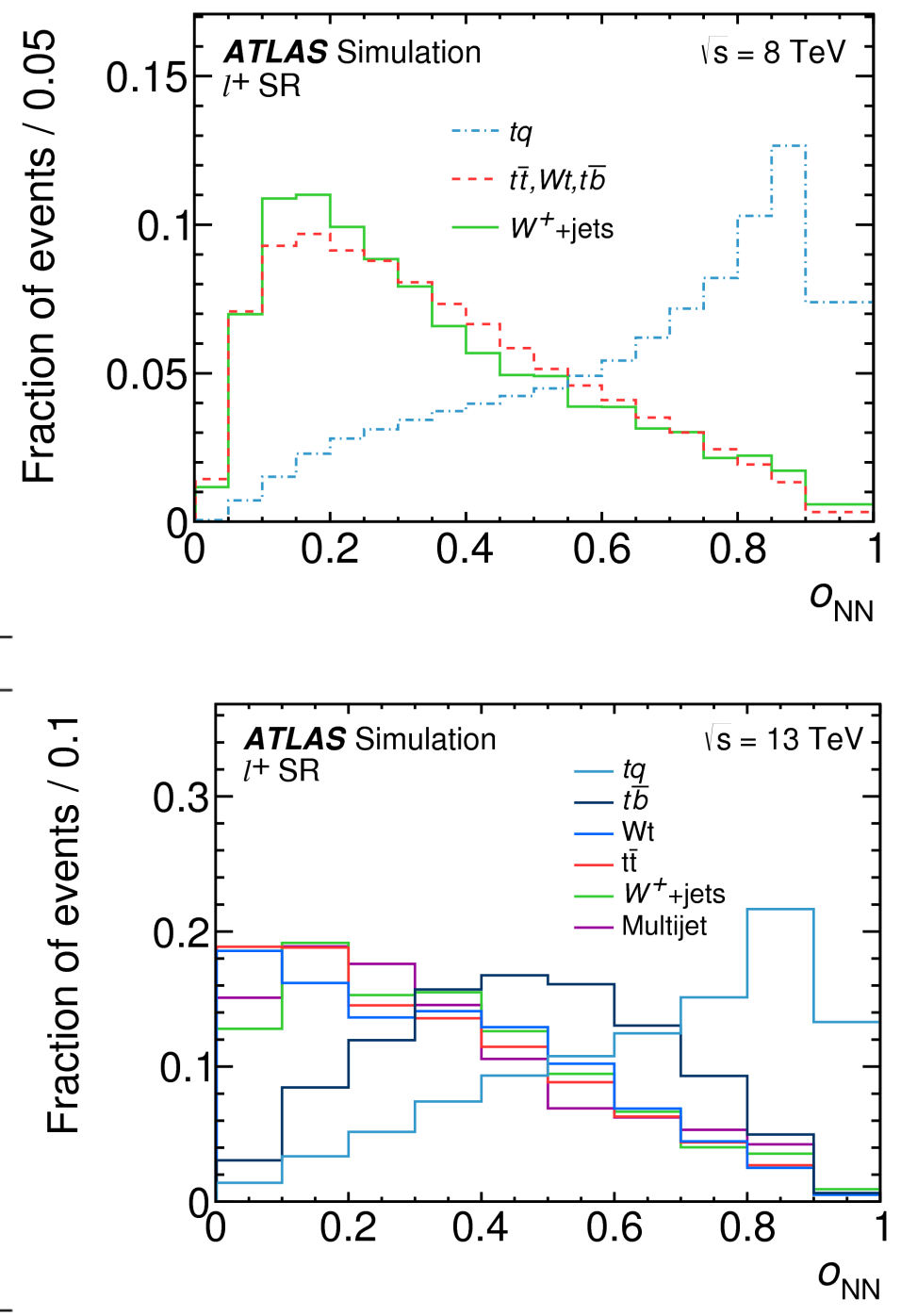